\title{
Symbol-Level Precoding Design Based on Distance Preserving Constructive Interference Regions
}

\author{
Alireza Haqiqatnejad, Student Member, IEEE, Farbod Kayhan, and Björn Ottersten, Fellow, IEEE
}

\begin{abstract}
In this paper, we investigate the symbol-level precoding (SLP) design problem in the downlink of a multiuser multipleinput single-output (MISO) channel. We consider generic twodimensional constellations with any shape and size, and confine ourselves to one of the main categories of constructive interference regions (CIR), namely, distance preserving CIR (DPCIR). We provide a comprehensive study of DPCIRs and derive several properties for these regions. Using these properties, we first show that any signal in a given DPCIR has a norm greater than or equal to the norm of the corresponding constellation point if and only if the convex hull of the constellation contains the origin. It is followed by proving that the power of the noise-free received signal in a DPCIR is a monotonic strictly increasing function of two parameters relating to the infinite Voronoi edges. Using the convex description of DPCIRs and their characteristics, we formulate two design problems, namely, the SLP power minimization with signal-to-interference-plus-noise ratio (SINR) constraints, and the SLP SINR balancing problem under max-min fairness criterion. The SLP power minimization based on DPCIRs can straightforwardly be written as a quadratic programming (QP). We derive a simplified reformulation of this problem which is less computationally complex. The SLP maxmin SINR, however, is non-convex in its original form, and hence difficult to tackle. We propose alternative optimization approaches, including semidefinite programming (SDP) formulation and block coordinate descent (BCD) optimization. We discuss and evaluate the loss due to the proposed alternative methods through extensive simulation results.
\end{abstract}

Index Terms-Distance preserving constructive interference region, downlink multiuser MISO, power minimization, SINR balancing, symbol-level precoding.

\section{INTRODUCTION}

Multiuser interference (MUI) is one of the major performance limiting factors in the downlink of multiuser systems which may adversely affect the achievable transmission rate of individual users. One approach to mitigate MUI is to precompensate for its undesired effect on the received signal through some signal processing at the transmitter, which is commonly known as multiuser precoding [1]. In general, (objective-oriented) multiuser precoding design can be expressed as a constrained optimization problem [2], [3]. The design problem aims at keeping a balance between some system-centric and user-centric objectives/requirements, depending on the network's operator strategy. Power and sumrate are often regarded as system-centric criteria [4]. Transmit

The authors are with Interdisciplinary Centre for Security, Reliability and Trust (SnT), University of Luxembourg, L-1855 Luxembourg. Email: \{alireza.haqiqatnejad,farbod.kayhan,bjorn.ottersten\}@uni.lu.

Part of this work has been presented in the $19^{\text {th }}$ IEEE International Workshop on Signal Processing Advances in Wireless Communications (SPAWC), Kalamata, Greece, 2018. power is considered, for example, to control the inter-cell interference in multicell wireless networks, and sum-rate is a measure of the overall system performance. On the other hand, as a user-centric criterion, signal-to-interference-plusnoise ratio (SINR) is an effective measure of quality-of-service (QoS) in multiuser interference channels [5]. In particular, both bit error rate (BER) and capacity, which are two relevant criteria from a practical point of view, are closely related with maximizing SINR [6]. Considering different types of optimization criteria, some well-known formulations for the multiuser precoding problem are QoS-constrained power minimization [7], [8], SINR balancing [6], [9], [10], and (weighted) sum-rate maximization [4], [11], [12]. In this paper, we primarily focus on the power minimization problem with SINR constraints and the SINR balancing problem using max-min fairness criterion.

Conventional multiuser precoding techniques try to exploit the knowledge of the channel in order to suppress MUI. A crucial assumption is therefore the availability of instantaneous or stochastic channel state information (CSI) at the transmitter [13]. However, MUI may not always considered to be harmful; on the contrary, following the notion of constructive interference (CI) [14], one can turn MUI into a useful source of signal power instead of treating it as an unwanted distortion [15]. To gain benefit from the potential advantage of CI, it has been recently suggested to design the precoder on a symbol-level basis as a promising alternative to linear block-level precoding [16]-[18]. Such a design concept is referred to as symbol-level precoding (SLP). Besides CSI, the symbol-level design also requires the instantaneous data information (DI) of all users which is readily available at the transmitter. When compared to conventional schemes, it has been shown that significant gains can be achieved at the expense of higher transmitter complexity [16], but without re-designing the receiver. While the linear structure of the precoder can be preserved under SLP, one may also form a virtual multicast formulation to directly find the optimal transmit vector, as proposed in [18], instead of designing the precoder matrix.

The symbol-level design of a multiuser precoder generally involves an optimization problem for each possible combination of the users' data symbols. The optimization constraints are so defined to push each user's (noise-free) received signal into a predefined region, called constructive interference region (CIR), enhancing (or guaranteeing a certain level of) detection accuracy. Therefore, formulation of the optimization problem, and particularly the constraints, depend on the adopted modulation scheme (i.e. signal constellation). The objective function, on the other hand, depends on the design criterion. 
The SLP problem minimizing the total transmit power has been studied for various constellations, including PSK [17][20], QAM [21], [22], and APSK [23]. For PSK constellations, the minimization of peak per-antenna transmit power is addressed in [24]. A generic formulation for power minimization problem, not depending on the constellation set, is presented in [25] for both total and peak per-antenna power constraints.

SINR balancing in multiuser multiple-input single-output (MISO) channels is generally more challenging and has been widely investigated for conventional precoding schemes (see e.g. [5], [6], [9], [26], [27]). This problem has been addressed in both multicast (single data stream) and unicast (independent data streams) downlink scenarios. The problem is not convex in general and is known to be NP-hard [5]; therefore, several alternate optimization approaches have been proposed in the literature. In particular, for downlink unicast channels, it is shown in [6] that the power minimization and the max-min SINR are inverse problems. We kindly refer the readers to [28] for a short review on SINR balancing in conventional multiuser precoding.

The SINR balancing problem for SLP schemes has not been addressed extensively in the literature. In [18], the nonconvex SLP max-min SINR is solved using its relation to the power minimization via a bisection search. The method is only applicable to PSK constellations (more precisely, to constant envelope modulations) and suffers from high computational complexity. This problem is also addressed in [17] and a second-order cone programming (SOCP) formulation is proposed for PSK constellations. Nevertheless, there is no general solution method or convex formulation for the SLP max-min SINR problem being valid for all generic (two-dimensional) constellation sets.

In this paper, we address both the power minimization and the SINR balancing problems in SLP. The proposed formulations will be shown to provide performance gains compared to the current schemes, with relatively reduced complexities. In defining the CI constraints, we specifically focus on a general family of CIRs, namely, distance preserving CIR (DPCIR), which has been previously introduced in [25]. This type of CIRs, along with its relaxed versions, are formally defined in [25]. We first elaborate the previous work by fully characterizing the geometry of DPCIRs based on the Voronoi regions of the constellation set. We also prove several properties for these regions which will subsequently be used to address the SLP design problems. The main property states that the norm of any signal in a given unbounded DPCIR is a monotonically increasing function of two parameters related to the corresponding infinite Voronoi edges, under the necessary and sufficient condition that the convex hull of the constellation contains the origin. We then study the SLP design criterion from a system-level point of view and discuss the feasibility of QoS provisioning in a resource-constrained multiuser downlink channel through deriving a sufficient feasibility condition. Furthermore, using the properties of DPCIRs, we show that by fixing a subset of variables in the optimization problem, the SLP max-min SINR can be treated as a convex problem. We also propose more tractable (but less computationally demanding) alternative optimization approaches, which results in competitive sub-optimal solutions for the original problem. Two methods are proposed and evaluated, namely, semidefinite programming (SDP) formulation and block coordinate descent (BCD) optimization. In summary, the main contributions of this paper are as follows:

1) We derive several properties for DPCIRs in order to further improve the SLP techniques and simplify the involved optimization problems.

2) We obtain a simple feasibility condition for the SLP power minimization problem, which is of practical importance in a realistic scenario as it determines whether the power minimization problem is applicable or not.

3) By rearranging the DPCIR-based SLP power minimization, we obtain an equivalent formulation with a reduced problem size.

4) We propose alternative (sub-optimal) solution methods for the SLP max-min SINR problem enhancing the performance of the multiuser system in terms of the worstuser SINR compared to the existing schemes, while being less computationally complex.

5) All the definitions and optimization problems are provided in a general form for (two-dimensional) constellation sets which are indifferent to both the shape and the order of constellation.

The remainder of this paper is organized as follows. In Section II, we describe our system model and define the problems of interest. In Section III, DPCIRs are explained and characterized for an arbitrary constellation set. This is followed by proving various properties for these regions. We address the SLP design problems in Section IV. Discussions on the power minimization problem and the proposed alternatives for SINR balancing are also included in this section. In Section V, we present our simulation results. Finally, we conclude the paper in Section VI.

The following notations are used in the rest of this paper. We use uppercase and lowercase bold-faced letters to denote matrices and vectors respectively, and italic letters to denote scalars. For matrices and vectors, $[\cdot]^{\mathrm{H}}$ and $[\cdot]^{\mathrm{T}}$ denote conjugate transpose and transpose operators, respectively. For vectors, $\|\cdot\|_{2}$ and $\|\cdot\|_{\infty}$ represent the $l_{2}$ norm and the $l_{\infty}$ norm, and $\succeq$ (or $\succ$ ) denotes componentwise inequality. For any vector $\mathbf{v}, \operatorname{diag}(\mathbf{v})$ represents a square matrix with $\mathbf{v}$ on its main diagonal and zero off-diagonal entries. blkdiag $(\cdot)$ represents a square block matrix having main-diagonal block matrices and zero off-diagonal blocks. Operators $|\cdot|, \Re\{\cdot\}, \Im\{\cdot\}$ and $(\cdot)^{*}$ respectively denote the amplitude, real part, imaginary part and conjugate of a complex input. Symbols $\mathbf{0 , 1}$ and I stand for allzeros vector, all-ones vector and identity matrix of appropriate dimensions. For any set $\mathcal{A},|\mathcal{A}|$ denotes the cardinality of $\mathcal{A}$. For any two sets (or vectors) $\mathcal{A}$ and $\mathcal{B}, \mathcal{A} \backslash \mathcal{B}$ denotes all the elements in $\mathcal{A}$ excluding those that are common with $\mathcal{B}$. $\mathbb{R}$ and $\mathbb{C}$ represent the sets of real and complex numbers, and $\mathbb{R}_{+}$and $\mathbb{R}_{++}$represent the sets of non-negative and positive real numbers, respectively. And $\mathbb{E}_{\mathrm{t}}\{\cdot\}$ denotes the expectation over symbol time. 


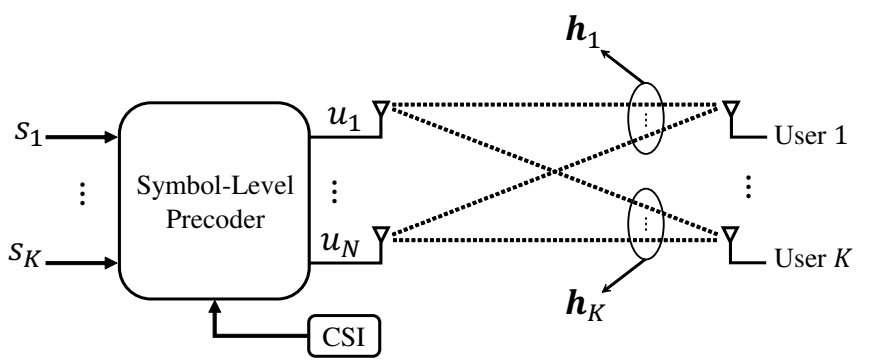

Fig. 1. SLP-based diagram of a downlink multiuser MISO unicast channel. The transmit signal vector $\mathbf{u}$, which is the instantaneous output of the symbollevel precoder, implicitly contains the data symbols of all the users.

\section{System Model and Problem Definition}

We consider the downlink of a multiuser MISO unicast channel, where a single base station (BS) sends independent data streams to $K$ users. The BS is equipped with $N$ transmit antennas while each user has a single receive antenna. As we will see in later sections, the number of users $K$ needs to not exceed the number of BS's antennas, i.e., $K \leq N$. A block fading channel is assumed between the BS's transmit antennas and the $k$-th user, where the complex channel vector is denoted by $\mathbf{h}_{k} \in \mathbb{C}^{1 \times N}$. It is further assumed that perfect channel knowledge is available at the BS.

At a given symbol time, $K$ independent data symbols are intended to be transmitted to $K$ users (throughout the paper, we drop the symbol's time index to simplify the notation). We collect these symbols in a vector $\mathbf{s}=\left[s_{1}, \ldots, s_{K}\right]^{\mathrm{T}} \in \mathbb{C}^{K \times 1}$ with $s_{k}$ denoting the symbol intended for the $k$-th user. Each symbol $s_{k}$ is drawn from a finite equiprobable twodimensional constellation set. Without loss of generality, we assume an $M$-ary constellation set $\mathcal{X}=\left\{x_{i} \mid x_{i} \in \mathbb{C}\right\}_{i=1}^{M}$ with unit average power for all the users. The user's symbol vector $\mathbf{s}$ is mapped to $N$ transmit antennas. This is done by a symbol-level precoder, yielding the transmit vector $\mathbf{u}=$ $\left[u_{1}, \ldots, u_{N}\right]^{\mathrm{T}} \in \mathbb{C}^{N \times 1}$, which (implicitly) contains the data symbols $s_{1}, \ldots, s_{K}$, as depicted in Fig. 1. The users' received signals are then given by

$$
r_{k}=\mathbf{h}_{k} \mathbf{u}+w_{k}, k=1, \ldots, K,
$$

where $w_{k} \sim \mathcal{C N}\left(0, \sigma_{k}^{2}\right)$ denotes the complex additive white Gaussian noise at the $k$-th receiver. From the received scalar $r_{k}$, the user $k$ may detect its own symbol $s_{k}$ by applying the single-user maximum-likelihood (ML) decision rule. Notice that the structure of the users' receivers is not affected by employing the symbol-level precoder at the transmitter.

\section{A. Constructive Interference Constraints}

The DI exploitation in symbol-level design is realized by processing the transmit signal in order to have the (noise-free) received signal of each user in a specific CIR associated with the desired symbol. CIRs are generally defined as the regions that preserve or enhance the symbol detection accuracy with respect to the original constellation set (see e.g. [15] and [25]).

In a downlink multiuser MISO scenario, the SINR of each user is conventionally defined as the ratio between the desired received signal power and the power of interfering components (due to the coexistence of other users) plus noise power. On the other hand, the SLP design generally aims at forcing all the received signal components to constructively interfere at the receiver of each user. This can be interpreted as having no destructive interference at none of the receivers, i.e., SINR boils down to signal-to-noise ratio (SNR) with CI included in the desired signal power. In other words, in the context of SLP, SINR is in fact signal-plus-interference-to-noise ratio and hence equivalent to conventional SNR. Nevertheless, in the rest of this paper we continue to use "SINR", as it has been commonly used in the literature on conventional and symbollevel multiuser precoding. More formally, it follows from (1) that the instantaneous received SINR of the $k$-th user at a given symbol instant is equal to

$$
\operatorname{SINR}_{k}=\frac{\mathbf{u}^{\mathrm{H}} \mathbf{h}_{k}^{\mathrm{H}} \mathbf{h}_{k} \mathbf{u}}{\sigma_{k}^{2}} .
$$

The user-specific requirements in a multiuser system are individual SINR thresholds that guarantee the reliable communication for all the users. It should, however, be noted that the given SINR thresholds typically refer to long-term (e.g. frame-level) SINRs, i.e., the average received SINR over all the symbols in a frame. Therefore, based on the instantaneous SINRs in (2), the following average SINR constraints are imposed on the design problem

$$
\mathbb{E}_{\mathrm{t}}\left\{\mathrm{SINR}_{k}\right\} \geq \gamma_{k}, k=1, \ldots, K,
$$

where $\gamma_{k}$ is the required SINR for the $k$-th user, and the expectations are taken over the entire frame (notice that while the time index is dropped for notational simplicity, the transmit vector $\mathbf{u}$ is a function of the symbol time). By substituting (2) for the instantaneous SINRs, (3) is equivalent to

$$
\mathbb{E}_{\mathrm{t}}\left\{\mathbf{u}^{\mathrm{H}} \mathbf{h}_{k}^{\mathrm{H}} \mathbf{h}_{k} \mathbf{u}\right\} \geq \sigma_{k}^{2} \gamma_{k}, k=1, \ldots, K .
$$

For sufficiently large frames (which is often the case in practice), we have $\mathbb{E}_{\mathrm{t}}\left\{s_{k}^{*} s_{k}\right\} \rightarrow 1, k=1, \ldots, K$. Hence, the average SINR constraints in (4) are satisfied if

$$
\mathbf{u}^{\mathrm{H}} \mathbf{h}_{k}^{\mathrm{H}} \mathbf{h}_{k} \mathbf{u} \geq \sigma_{k}^{2} \gamma_{k} s_{k}^{*} s_{k}, k=1, \ldots, K .
$$

One can think of the symbol-level constraints in (5) as a conservative way to meet the average SINR requirements.

\section{B. Problem Definition}

The functionality of SLP is to instantaneously design the signal to be transmitted in each symbol time based on a CI-constrained optimization problem. The solution of this problem, i.e., the transmit vector $\mathbf{u}$, is in general a function of instantaneous DI and CSI as well as a set of given system constraints or user-specific requirements.

For each user $k$, the noise-free received signal is pushed by the precoder to be inside the corresponding CIR up to a scale factor that depends on the given SINR requirement. Accordingly, the SLP power minimization problem being constrained by CIRs (for a generic constellation) as well as individual SINR thresholds can be formulated as

$$
\begin{aligned}
\underset{\mathbf{u}}{\operatorname{minimize}} & f(\mathbf{u}) \\
\text { s.t. } & \mathbf{h}_{k} \mathbf{u} \in \sigma_{k} \sqrt{\gamma_{k}} \mathcal{D}_{k}, k=1, \ldots, K,
\end{aligned}
$$


where $\mathcal{D}_{k}$ represents the CIR associated with symbol $s_{k}$, and is typically interpreted in a way that it pushes $\mathbf{h}_{k} \mathbf{u}$ away from the corresponding decision boundaries (deeper into the decision region of $s_{k}$ ). An explicit definition for $\mathcal{D}_{k}$, which in general depends on the type of CIR, will be provided in the next section by adopting the specific case of DPCIRs. The objective function $f(\mathbf{u})$ in (6) can be either $\mathbf{u}^{\mathrm{H}} \mathbf{u}$ or $\|\mathbf{u}\|_{\infty}^{2}$, depending on whether the total or the peak (per-antenna) transmit power is minimized. It is important to notice that a sufficient (but not necessary) condition under which the optimal solution of (6) satisfies the SINR constraints in (5) is that the amplitude of any point in $\mathcal{D}_{k}$ is at least equal to $\left|s_{k}\right|=\sqrt{s_{k}^{*} s_{k}}$, for all $k=1, \ldots, K$, i.e.,

$$
\sigma_{k}^{2} \gamma_{k} x^{*} x \geq \sigma_{k}^{2} \gamma_{k} s_{k}^{*} s_{k}, \forall x \in \mathcal{D}_{k} .
$$

The SLP SINR balancing problem, on the other hand, aims to serve all the users in a fair manner with a given system-centric restriction (usually the total transmit power). In particular, under the max-min fairness criterion, the goal is to maximize the worst SINR among all users subject to a total power constraint. This leads to the following formulation:

$$
\begin{aligned}
\underset{\mathbf{u}}{\operatorname{maximize}} & \min _{k}\left\{\frac{\mathbf{u}^{\mathrm{H}} \mathbf{h}_{k}^{\mathrm{H}} \mathbf{h}_{k} \mathbf{u}}{\sigma_{k}^{2}}\right\}_{k=1}^{K} \\
\text { s.t. } & \mathbf{h}_{k} \mathbf{u} \in \sigma_{k} \mathcal{D}_{k}, k=1, \ldots, K, \\
& \mathbf{u}^{\mathrm{H}} \mathbf{u} \leq P,
\end{aligned}
$$

where $P$ is the downlink total power budget. It should be noted that in practice, $P$ may be given as the average power budget for the downlink transmission, while the power constraint in (8) controls the instantaneous total transmit power in each symbol time. This is a sufficient constraint to meet the average power budget, but clearly it is not necessary and has been considered in order to simplify the problem.

We will reformulate and discuss both problems (6) and (8) in Section IV, assuming the CIRs to be distance preserving. To this end, we first present a detailed study of the DPCIRs which enables us to exploit their properties in order to properly form the constraints of the SLP optimization problems.

\section{Distance Preserving Constructive INTERFERENCE REGIONS}

In this section, we study a general category of CIRs, namely, DPCIRs, and develop their characterizations by deriving some of their properties. The main results of this section are stated in Lemma 2, Lemma 3 and Theorem 1. The proofs have been previously presented in [28]. For the sake of completeness, we provide the proofs also in this paper in appendices A-C. Hereafter, we denote each complex-valued constellation point by its equivalent real-valued vector notation, hence the set of points in $\mathcal{X}$ is denoted by $\left\{\mathbf{x}_{i} \mid \mathbf{x}_{i} \in \mathbb{R}^{2}\right\}_{i=1}^{M}$.

DPCIRs have been introduced in [25] as type of CIRs that preserve the Euclidean distances between the constellation points, i.e., they do not increase the symbol error probabilities of the users. In other words, any point from a particular DPCIR (which corresponds to one of the constellation points) has an increased distance to all the other constellation points in $\mathcal{X}$. In the sequel, a systematic representation of DPCIRs based on the ML decision regions of the constellation set $\mathcal{X}$ is provided, which will help us to further study their characteristics.

For the assumed equiprobable constellation set $\mathcal{X}$, the $\mathrm{ML}$ decision rule corresponds to the Voronoi regions of $\mathcal{X}$ which are bounded by hyperplanes. For a given constellation point $\mathbf{x}_{i}$ and one of its neighboring points $\mathbf{x}_{j}$ (the neighboring points are referred to those points that share an ML decision boundary with $\mathbf{x}_{i}$ ), the hyperplane separating the Voronoi regions of $\mathbf{x}_{i}$ and $\mathbf{x}_{j}$ is given by $\left\{\mathbf{x} \mid \mathbf{x} \in \mathbb{R}^{2}, \mathbf{a}_{i, j}^{\mathrm{T}} \mathbf{x}=b_{i, j}\right\}$, where $\mathbf{a}_{i, j}=$ $\mathbf{x}_{i}-\mathbf{x}_{j}$ (or any non-zero scalar multiplication of $\mathbf{x}_{i}-\mathbf{x}_{j}$ ), and $b_{i, j}=\frac{1}{2} \mathbf{a}_{i, j}^{\mathrm{T}}\left(\mathbf{x}_{i}+\mathbf{x}_{j}\right)$. This hyperplane indicates the ML decision boundary (Voronoi edge) between $\mathbf{x}_{i}$ and $\mathbf{x}_{j}$, which splits $\mathbb{R}^{2}$ plane into two halfspaces (notice that hyperplanes are infinite lines in $\mathbb{R}^{2}$ ). The closed halfspace that contains the decision region of $\mathbf{x}_{i}$ is represented as

$$
\mathcal{H}_{i, j, \mathrm{ML}}=\left\{\mathbf{x} \mid \mathbf{x} \in \mathbb{R}^{2}, \mathbf{a}_{i, j}^{\mathrm{T}} \mathbf{x} \geq b_{i, j}\right\},
$$

where $\mathbf{a}_{i, j}$ is the inward normal and $b_{i, j}$ determines the offset from the origin. The Voronoi region of $\mathbf{x}_{i}$ is then given by intersecting the halfspaces in the form of (9) over all the neighboring points of $\mathbf{x}_{i}$, i.e.,

$$
\begin{aligned}
\mathcal{D}_{i, \mathrm{ML}} & =\bigcap_{j \in \mathcal{J}_{i}} \mathcal{H}_{i, j, \mathrm{ML}} \\
& =\left\{\mathbf{x} \mid \mathbf{x} \in \mathbb{R}^{2}, \mathbf{a}_{i, j}^{\mathrm{T}} \mathbf{x} \geq b_{i, j}, \forall j \in \mathcal{J}_{i}\right\},
\end{aligned}
$$

where $\mathcal{J}_{i}=\left\{j \mid \mathbf{x}_{j} \in \mathcal{S}_{i}\right\}$ and $\mathcal{S}_{i}$ denotes the set of neighboring points of $\mathbf{x}_{i}$, i.e., the set of points having a common decision boundary with $\mathbf{x}_{i}$, with $\left|\mathcal{S}_{i}\right|=\left|\mathcal{J}_{i}\right|=M_{i}$. It can be easily shown that a Voronoi regions is always a convex set and can be either an unbounded or bounded polyhedron [29], depending on the relative geometry of $\mathbf{x}_{i}$ in $\mathcal{X}$. The Voronoi region (10) can be expressed in a more compact form as

$$
\mathcal{D}_{i, \mathrm{ML}}=\left\{\mathbf{x} \mid \mathbf{x} \in \mathbb{R}^{2}, \mathbf{A}_{i} \mathbf{x} \succeq \mathbf{b}_{i}\right\},
$$

where $\mathbf{A}_{i} \in \mathbb{R}^{M_{i} \times 2}$ and $\mathbf{b}_{i} \in \mathbb{R}^{M_{i}}$ contain $\mathbf{a}_{i, j}^{\mathrm{T}}$ and $b_{i, j}$, respectively, for all $j \in \mathcal{J}_{i}$. The halfspace representation of the ML decision regions in (11) can be used to describe the DPCIRs [25], as will be explained in the following.

The distance preserving margin between $\mathbf{x}_{i}$ and $\mathbf{x}_{j}$, by definition, is equal to $\frac{1}{2} d_{i, j}$, where $d_{i, j}=\left\|\mathbf{x}_{i}-\mathbf{x}_{j}\right\|_{2}$ denotes the Euclidean distance between $\mathbf{x}_{i}$ and $\mathbf{x}_{j}$. Accordingly, given the Voronoi hyperplane $\left\{\mathbf{x} \mid \mathbf{x} \in \mathbb{R}^{2}, \mathbf{a}_{i, j}^{\mathrm{T}} \mathbf{x}=b_{i, j}\right\}$, the corresponding distance preserving hyperplane can be represented by $\left\{\mathbf{x} \mid \mathbf{x} \in \mathbb{R}^{2}, \mathbf{a}_{i, j}^{\mathrm{T}} \mathbf{x}=b_{i, j}+c_{i, j}\right\}$, where $c_{i, j}=\frac{1}{2} d_{i, j}\left\|\mathbf{a}_{i, j}\right\|_{2}$. These two hyperplanes are parallel to each other with an orthogonal distance of $\frac{c_{i, j}}{\left\|\mathbf{a}_{i, j}\right\|_{2}}$ in the direction of $\mathbf{a}_{i, j}$. The resulting closed halfspace is then given by

$$
\mathcal{H}_{i, j, \mathrm{DP}}=\left\{\mathbf{x} \mid \mathbf{x} \in \mathbb{R}^{2}, \mathbf{a}_{i, j}^{\mathrm{T}} \mathbf{x} \geq b_{i, j}+c_{i, j}\right\} .
$$

Intersecting (12) over all the neighboring points of $\mathbf{x}_{i}$ gives the associated DPCIR as

$$
\begin{aligned}
\mathcal{D}_{i, \mathrm{DP}} & =\bigcap_{j \in \mathcal{J}_{i}} \mathcal{H}_{i, j, \mathrm{DP}} \\
& =\left\{\mathbf{x} \mid \mathbf{x} \in \mathbb{R}^{2}, \mathbf{a}_{i, j}^{\mathrm{T}} \mathbf{x} \geq b_{i, j}+c_{i, j}, \forall j \in \mathcal{J}_{i}\right\},
\end{aligned}
$$

which can be written in the matrix form as

$$
\mathcal{D}_{i, \mathrm{DP}}=\left\{\mathbf{x} \mid \mathbf{x} \in \mathbb{R}^{2}, \mathbf{A}_{i} \mathbf{x} \succeq \mathbf{b}_{i}+\mathbf{c}_{i}\right\},
$$


where $\mathbf{c}_{i} \in \mathbb{R}_{+}^{M_{i}}$ is the vector containing $\frac{1}{2} d_{i, j}\left\|\mathbf{a}_{i, j}\right\|_{2}$ for all $j \in \mathcal{J}_{i}$. Similar to $\mathcal{D}_{i, \mathrm{ML}}$, the region $\mathcal{D}_{i, \mathrm{DP}}$ is obtained by the intersection of a number of closed halfspaces, and thus is a polyhedron. Furthermore, the bounding hyperplanes of $\mathcal{D}_{i, \mathrm{DP}}$ are parallel to their corresponding Voronoi edges, i.e., they have the same inward normals $\mathbf{a}_{i, j}, j \in \mathcal{J}_{i}$.

Remark 1. Matrix $\mathbf{A}_{i}$ and vectors $\mathbf{b}_{i}$ and $\mathbf{c}_{i}$ uniquely describe $\mathcal{D}_{i, \mathrm{DP}}$ associated with the $i$-th constellation point. By definition, $\mathbf{A}_{i}$ is the matrix that contains the normal vectors of DPCIR boundaries (hyperplanes), given by

$$
\mathbf{A}_{i}=\left[\begin{array}{c}
\mathbf{a}_{i, 1}^{\mathrm{T}} \\
\vdots \\
\mathbf{a}_{i, M_{i}}^{\mathrm{T}}
\end{array}\right]=\left[\begin{array}{c}
\left(\mathbf{x}_{i}-\mathbf{x}_{i, 1}\right)^{\mathrm{T}} \\
\vdots \\
\left(\mathbf{x}_{i}-\mathbf{x}_{i, M_{i}}\right)^{\mathrm{T}}
\end{array}\right],
$$

and vectors $\mathbf{b}_{i}$ and $\mathbf{c}_{i}$ respectively contain the offsets from the origin and from the ML decision boundaries, i.e.,

$$
\begin{gathered}
\mathbf{b}_{i}=\left[\begin{array}{c}
\frac{1}{2} \mathbf{a}_{i, 1}^{\mathrm{T}}\left(\mathbf{x}_{i}+\mathbf{x}_{i, 1}\right) \\
\vdots \\
\frac{1}{2} \mathbf{a}_{i, M_{i}}^{\mathrm{T}}\left(\mathbf{x}_{i}+\mathbf{x}_{i, M_{i}}\right)
\end{array}\right], \\
\mathbf{c}_{i}=\left[\begin{array}{c}
\frac{1}{2} d_{i, 1}\left\|\mathbf{a}_{i, 1}\right\|_{2} \\
\vdots \\
\frac{1}{2} d_{i, M_{i}}\left\|\mathbf{a}_{i, M_{i}}\right\|_{2}
\end{array}\right]=\left[\begin{array}{c}
\frac{1}{2}\left\|\mathbf{x}_{i}-\mathbf{x}_{i, 1}\right\|_{2}^{2} \\
\vdots \\
\frac{1}{2}\left\|\mathbf{x}_{i}-\mathbf{x}_{i, M_{i}}\right\|_{2}^{2}
\end{array}\right],
\end{gathered}
$$

where $\mathbf{x}_{i, 1}, \ldots, \mathbf{x}_{i, M_{i}} \in \mathcal{S}_{i}$ denote the neighboring constellation points of $\mathbf{x}_{i}$ and $d_{i, j}=\left\|\mathbf{x}_{i}-\mathbf{x}_{i, j}\right\|_{2}, j \in \mathcal{J}_{i}$ is the Euclidean distance between $\mathbf{x}_{i}$ and $\mathbf{x}_{i, j}$. After simple algebraic steps on (16) and (17), we obtain

$$
\mathbf{b}_{i}+\mathbf{c}_{i}=\left[\begin{array}{c}
\left(\mathbf{x}_{i}-\mathbf{x}_{i, 1}\right)^{\mathrm{T}} \mathbf{x}_{i} \\
\vdots \\
\left(\mathbf{x}_{i}-\mathbf{x}_{i, M_{i}}\right)^{\mathrm{T}} \mathbf{x}_{i}
\end{array}\right] .
$$

Using (15) and (18), the region $\mathcal{D}_{i, \mathrm{DP}}$ in (14) can be simply expressed as

$$
\mathcal{D}_{i, \mathrm{DP}}=\left\{\mathbf{x} \mid \mathbf{x} \in \mathbb{R}^{2}, \mathbf{A}_{i}\left(\mathbf{x}-\mathbf{x}_{i}\right) \succeq \mathbf{0}\right\},
$$

which describes $\mathcal{D}_{i, \mathrm{DP}}$ as a vector space originated at $\mathbf{x}_{i}$ and (non-negatively) spanned by the row vectors of $\mathbf{A}_{i}$.

It is straightforward to show that the following properties hold for DPCIRs:

Property 1. For all $\mathrm{x}_{i} \in \mathcal{X}$ and any $\mathrm{x} \in \mathcal{D}_{i, \mathrm{DP}}$, we have

i. $\mathcal{D}_{i, \mathrm{DP}} \subseteq \mathcal{D}_{i, \mathrm{ML}}$.

ii. $\|\mathbf{x}-\mathbf{y}\|_{2} \geq\left\|\mathbf{x}_{i}-\mathbf{x}_{j}\right\|_{2}=d_{i, j}, \forall \mathbf{x}_{j} \in \mathcal{X}, \forall \mathbf{y} \in \mathcal{D}_{j, \mathrm{DP}}$.

As a special case of Property 1-ii for $\mathbf{y}=\mathbf{x}_{j}$, we have

$$
\left\|\mathbf{x}-\mathbf{x}_{j}\right\|_{2} \geq\left\|\mathbf{x}_{i}-\mathbf{x}_{j}\right\|_{2}, \forall \mathbf{x}_{j} \in \mathcal{X}
$$

where (20) holds with equality only when $\mathbf{x}=\mathbf{x}_{i}$.

The convex hull of $\mathcal{X}$, denoted by $\operatorname{conv} \mathcal{X}$, refers to the smallest convex set containing $\mathcal{X}$ and can be simply derived from the constellation set $\mathcal{X}$. The set of points belonging to the boundary of $\operatorname{conv} \mathcal{X}$ is denoted by $\mathbf{b d} \mathcal{X}$, and the set of interior points of $\operatorname{conv} \mathcal{X}$, i.e., $\operatorname{conv} \mathcal{X} \backslash \mathbf{b d} \mathcal{X}$, is denoted by int $\mathcal{X}$. A typical illustration of the aforementioned sets for the optimized 8 -ary constellation [30] is shown in Fig. 2 (a). It follows from (14) that if $\mathcal{D}_{i, \mathrm{ML}}$ is bounded, then $\mathcal{D}_{i, \mathrm{DP}}=\mathbf{x}_{i}$, which means

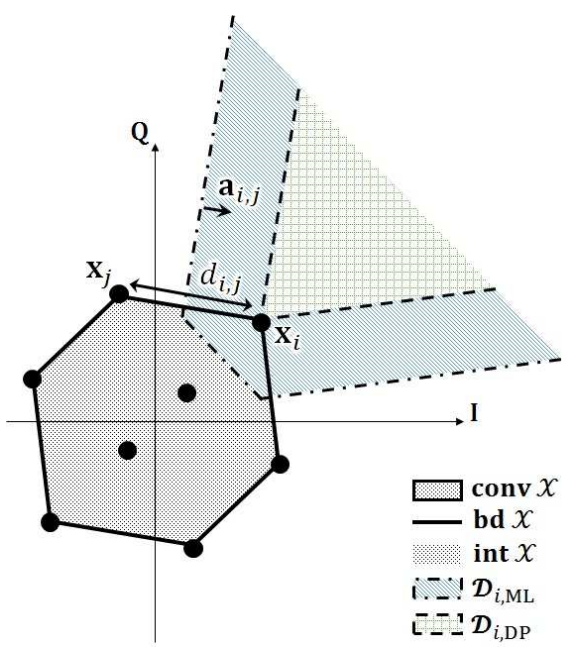

(a)

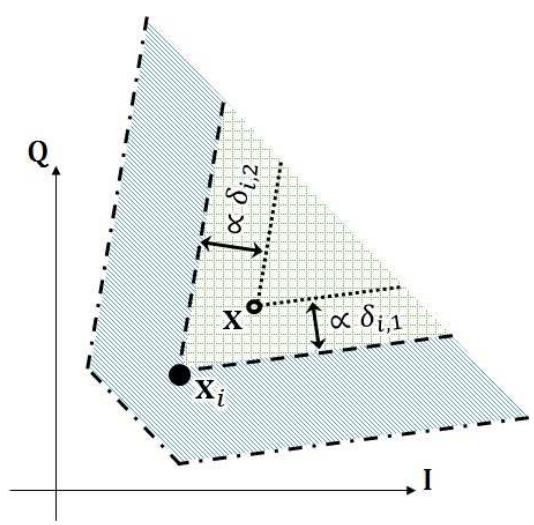

(b)

Fig. 2. The optimized 8-ary constellation. (a) A boundary points $\mathbf{x}_{i}$ with unbounded Voronoi region; $\mathcal{D}_{i, \mathrm{DP}}$ is a polyhedral angle with two infinite edges starting from $\mathbf{x}_{i}$. (b) Any point $\mathbf{x} \in \mathcal{D}_{i, \text { DP }}$ can be specified by (25) if one displaces the two infinite boundary hyperplanes, each of which by an orthogonal distance proportional to $\delta_{i, 1}$ or $\delta_{i, 2}$.

that all the inequalities in (14) are satisfied with equality. On the other hand, for an unbounded $\mathcal{D}_{i, \mathrm{ML}}$, the associated $\mathcal{D}_{i, \mathrm{DP}}$ is an unbounded polyhedron (more specifically, a polyhedral angle as depicted in Fig. 2 (a)) which is explicitly characterized using the two following lemmas.

Lemma 1. A point $\mathbf{x}_{i} \in \mathcal{X}$ lies on the boundary of (or is a vertex of) conv $\mathcal{X}$ iff its Voronoi region $\mathcal{D}_{i, \mathrm{ML}}$ is unbounded [31, Lemma 2.2].

Lemma 2. For every $\mathbf{x}_{i} \in \mathcal{X}$ with unbounded $\mathcal{D}_{i, M L}, \mathcal{D}_{i, D P}$ is a polyhedral angle with a vertex at $\mathbf{x}_{i}$, and each of its edges is perpendicular to one of the two line segments connecting $\mathbf{x}_{i}$ to its two neighboring points on $\mathbf{b d} \mathcal{X}$.

Proof: See Appendix A.

For any $\mathbf{x}_{i} \in \mathbf{b d} \mathcal{X}$, Lemma 2 implicitly states that $\mathcal{D}_{i, \mathrm{DP}}$ is not affected by changing the geometry of any point $\mathbf{x}_{j} \in$ int $\mathcal{X}$, as well as by adding a new constellation point on either $\mathbf{b d} \mathcal{X}$ or int $\mathcal{X}$. This is because the direction of $\mathbf{a}_{i, j}$ remains unchanged for all $\mathbf{x}_{j} \in \mathcal{S}_{i} \cap \mathbf{b d} \mathcal{X}$ under the above operations.

Next, we prove that the norm of any point in a DPCIR is always greater than or equal to the norm of the corresponding 
vertex if and only if the convex hull of the constellation includes the origin. It should be noted that this is a rather light condition, as all well-known constellations in the literature with $M \geq 4$ have at least one point in each quadrant and therefore their convex hull contains the origin.

Lemma 3. For any constellation point $\mathbf{x}_{i} \in \mathcal{X}$, we have $\|\mathbf{x}\| \geq\left\|\mathbf{x}_{i}\right\|, \forall \mathbf{x} \in \mathcal{D}_{i, \mathrm{DP}}$ iff $\operatorname{conv} \mathcal{X}$ contains the origin. Equality is achieved only when $\mathbf{x}=\mathbf{x}_{i}$.

Proof: See Appendix B.

To proceed, it is more convenient to re-express the linear inequalities in (14) by an equivalent set of linear equations. By introducing a non-negative vector $\boldsymbol{\delta}_{i}$, the region $\mathcal{D}_{i, \mathrm{DP}}$ can be equivalently described as

$$
\mathcal{D}_{i, \mathrm{DP}}=\left\{\mathbf{x} \mid \mathbf{x} \in \mathbb{R}^{2}, \mathbf{A}_{i} \mathbf{x}=\mathbf{b}_{i}+\mathbf{c}_{i}+\boldsymbol{\delta}_{i}, \boldsymbol{\delta}_{i} \in \mathbb{R}_{+}^{M_{i}}\right\},
$$

which will be used as the CI constraint in our formulations of the SLP optimization problem in the next section. The linear equations in (21) indicate that any $\mathrm{x} \in \mathcal{D}_{i, \mathrm{DP}}$ can be represented as the intersection point of $M_{i}$ hyperplanes, each of which is parallel to one of $M_{i}$ boundaries of $\mathcal{D}_{i, \mathrm{DP}}$ but has a different offset due to the vector $\boldsymbol{\delta}_{i}$.

Remark 2. As mentioned earlier, for an interior constellation point $\mathbf{x}_{i} \in \operatorname{int} \mathcal{X}$, we always have $\mathcal{D}_{i, \mathrm{DP}}=\mathbf{x}_{i}$, which is the unique solution of $\mathbf{A}_{i} \mathbf{x}=\mathbf{b}_{i}+\mathbf{c}_{i}$, i.e.,

$$
\mathcal{D}_{i, \mathrm{DP}}=\left\{\mathbf{x} \mid \mathbf{x} \in \mathbb{R}^{2}, \mathbf{A}_{i} \mathbf{x}=\mathbf{b}_{i}+\mathbf{c}_{i}\right\} .
$$

It then follows from (21) that

$$
\boldsymbol{\delta}_{i}=\mathbf{0}, \forall \mathbf{x}_{i} \in \operatorname{int} \mathcal{X}
$$

It can be easily verified that for any $\mathbf{x}_{i} \in \operatorname{int} \mathcal{X}$, the region $\mathcal{D}_{i, \mathrm{DP}}$ is bounded by $M_{i} \geq 3$ hyperplanes of which at least two are not parallel. This allows us to represent $\mathcal{D}_{i, \mathrm{DP}}$ as the intersection point of these two non-parallel hyperplanes by considering $\boldsymbol{\delta}_{i}=\mathbf{0}$. Consequently, $\mathbf{A}_{i}$ can be written as a $2 \times 2$ full rank matrix.

On the other hand, it is shown that a hyperplane in a set of hyperplanes describing the boundaries of a polyhedron is redundant if the corresponding polyhedron remains unchanged by removing that hyperplane [32, p. 9]. Therefore, in the rest we consider the minimal set of hyperplanes that are sufficient to describe $\mathcal{D}_{i, \mathrm{DP}}$ by removing from (21) the equalities that come from a redundant hyperplane. As a result, and based on Lemma 2 , for any $\mathbf{x}_{i} \in \mathbf{b d} \mathcal{X}$, the associated region $\mathcal{D}_{i, \mathrm{DP}}$ is spanned by two normal vectors corresponding to the (infinite) boundaries of $\mathcal{D}_{i \text {,DP }}$, i.e.,

$$
\mathcal{D}_{i, \mathrm{DP}}=\left\{\mathbf{x} \mid \mathbf{x} \in \mathbb{R}^{2}, \mathbf{A}_{i} \mathbf{x}=\mathbf{b}_{i}+\mathbf{c}_{i}+\boldsymbol{\delta}_{i}, \boldsymbol{\delta}_{i} \in \mathbb{R}_{+}^{2}\right\},
$$

from which any point $\mathbf{x} \in \mathcal{D}_{i, \mathrm{DP}}$ can be specified by two nonnegative coefficients as

$$
\boldsymbol{\delta}_{i}=\left[\delta_{i, 1}, \delta_{i, 2}\right]^{\mathrm{T}} \in \mathbb{R}_{+}^{2}, \forall \mathbf{x}_{i} \in \mathbf{b d} \mathcal{X},
$$

which makes $\mathbf{A}_{i}$ a $2 \times 2$ full rank (hence non-singular) matrix. It should be pointed out that this representation also covers the special case with the two infinite boundary hyperplanes being parallel to each other (e.g., QAM constellations). In such case, both $\delta_{i, 1}$ and $\delta_{i, 2}$ are constrained to be always zero. However, the region $\mathcal{D}_{i, \mathrm{DP}}$, which is a half-line starting from the constellation point $\mathbf{x}_{i}$, can be spanned by a non-negative scalar indicating the offset of a virtual hyperplane orthogonal to the two existing infinite boundaries (which preserves the non-singularity of $\mathbf{A}_{i}$ ). Thereby, any point $\mathbf{x} \in \mathcal{D}_{i, \mathrm{DP}}$ can be represented by $\boldsymbol{\delta}_{i}=\left[\delta_{i, 1}, 0\right]^{\mathrm{T}}, \delta_{i, 1} \in \mathbb{R}_{+}$.

It is also important to notice that our derivations above are presented for two-dimensional constellation sets. However, same concepts can be defined for both one-dimensional (e.g. $M$-PAM) and multi-dimensional (e.g. $M$-FSK) modulation schemes. In general, one may define

$$
\mathcal{D}_{i, \mathrm{DP}}=\left\{\mathbf{x} \mid \mathbf{x} \in \mathbb{R}^{m}, \mathbf{A}_{i} \mathbf{x}=\mathbf{b}_{i}+\mathbf{c}_{i}+\boldsymbol{\delta}_{i}, \boldsymbol{\delta}_{i} \in \mathbb{R}_{+}^{m}\right\},
$$

with $m$ denoting the dimensionality of the constellation set. In this general case, a number of $m$ normal vectors (each corresponding to a boundary hyperplane) is sufficient to span the entire region, i.e., any $\mathbf{x} \in \mathcal{D}_{i \text {,DP }}$ can be specified by an $m$-dimensional vector $\boldsymbol{\delta}_{i}$ as

$$
\boldsymbol{\delta}_{i}=\left[\delta_{i, 1}, \ldots, \delta_{i, m}\right]^{\mathrm{T}} \in \mathbb{R}_{+}^{m} .
$$

based on which $\mathbf{A}_{i}, \mathbf{b}_{i}$ and $\mathbf{c}_{i}$ in (26) are constructed with appropriate dimensions. In a special case of $M$-PAM (with one-dimensional DPCIRs), we have $\delta_{i}=\delta_{i} \in \mathbb{R}_{+}$which helps us to reduce the SLP max-min SINR to an equivalent convex optimization problem, as we will see in Section IV.

Finally, we state the following theorem which will be used to formulate the SLP design problems in the next section.

Theorem 1. For any constellation point $\mathbf{x}_{i} \in \mathbf{b d} \mathcal{X}$ with $\mathcal{D}_{i, \mathrm{DP}}$ as expressed in (21), function $f(\mathbf{x})=\|\mathbf{x}\|$ over its domain $\mathcal{D}_{i, \mathrm{DP}}$ is a monotonic strictly increasing function of each element of $\boldsymbol{\delta}_{i}$ iff $\operatorname{conv} \mathcal{X}$ contains the origin.

\section{Proof: See Appendix C}

It is worth noting that Theorem 1 can be further generalized for more generic CIRs, namely, union bound (UBCIR) and minimum distance preserving (MDPCIR) as defined in [25]. In both cases, the norm of any point belonging to these regions is strictly increasing in exactly two coefficients related to the two infinite boundary hyperplanes.

\section{Symbol-Level Precoding Design Problem}

In this section, by using the properties of DPCIRs proved in the previous section, we formulate the optimization problems of multiuser precoding on a symbol-level basis. In particular, we are interested in two well-known design problems, namely, power minimization and SINR balancing. As discussed in Section III, the DPCIRs can be explicitly obtained for all generic constellations as they depend only on the Voronoi regions. This enables us to arrange the optimization problems in a general form which is indifferent to the shape of constellation.

Throughout this section, for any user $k=1, \ldots, K$, the data symbol $s_{k}$ corresponds to one of the points $\left\{\mathbf{x}_{i}\right\}_{i=1}^{M}$ in $\mathcal{X}$. We denote by $i_{k}$ the index of the constellation point corresponding to $s_{k}$, i.e.,

$$
\left[\begin{array}{l}
\Re\left\{s_{k}\right\} \\
\Im\left\{s_{k}\right\}
\end{array}\right]=\mathbf{x}_{i_{k}}, i_{k} \in\{1, \ldots, M\} .
$$


Furthermore, we define the index set $\mathcal{K}=\left\{k \mid \mathbf{x}_{i_{k}} \in \mathbf{b d} \mathcal{X}\right\}$ referring to those users with a symbol on the boundary of $\mathcal{X}$. In the following, we rearrange vectors $\mathbf{u}$ and $\mathbf{h}_{k}$ as

$$
\begin{gathered}
\tilde{\mathbf{u}}=\left[\begin{array}{c}
\Re\{\mathbf{u}\} \\
\Im\{\mathbf{u}\}
\end{array}\right] \in \mathbb{R}^{2 N \times 1}, \\
\mathbf{H}_{k}=\left[\begin{array}{cc}
\Re\left\{\mathbf{h}_{k}\right\} & -\Im\left\{\mathbf{h}_{k}\right\} \\
\Im\left\{\mathbf{h}_{k}\right\} & \Re\left\{\mathbf{h}_{k}\right\}
\end{array}\right] \in \mathbb{R}^{2 \times 2 N}, k=1, \ldots, K,
\end{gathered}
$$

respectively, such that $\mathbf{H}_{k} \tilde{\mathbf{u}}$ represents the noise-free received signal at the $k$-th user's receiver. It is easy to check that $\mathbf{u}^{\mathrm{H}} \mathbf{u}=$ $\tilde{\mathbf{u}}^{\mathrm{T}} \tilde{\mathbf{u}}$. We also denote by

$$
\begin{gathered}
\mathbf{G}=\left[\begin{array}{c}
\mathbf{A}_{i_{1}} \mathbf{H}_{1} \\
\vdots \\
\mathbf{A}_{i_{K}} \mathbf{H}_{K}
\end{array}\right] \in \mathbb{R}^{2 K \times 2 N}, \mathbf{b}=\left[\mathbf{b}_{i_{1}}, \ldots, \mathbf{b}_{i_{K}}\right]^{\mathrm{T}} \in \mathbb{R}^{2 K}, \\
\mathbf{c}=\left[\mathbf{c}_{i_{1}}, \ldots, \mathbf{c}_{i_{K}}\right]^{\mathrm{T}} \in \mathbb{R}^{2 K}, \boldsymbol{\delta}=\left[\boldsymbol{\delta}_{i_{1}}, \ldots, \boldsymbol{\delta}_{i_{K}}\right]^{\mathrm{T}} \in \mathbb{R}^{2 K},
\end{gathered}
$$

the vectors and matrices collecting the CIR parameters for all $K$ users. Notice that, in general, the number of rows in matrix $\mathbf{G}$, as well as the number of entries in vectors $\mathbf{b}$ and $\mathbf{c}$, are equal to the summation of the number of neighboring constellation points of $\mathbf{x}_{i_{k}}$ over all $k=1, \ldots, K$, i.e., $\sum_{k=1}^{K} M_{i_{k}}$. However, as a consequence of (22)-(25) in Remark 2, only two hyperplanes (linear equations) are sufficient to entirely span $\mathcal{D}_{i_{k}, \text { DP }}$ for all $k=1, \ldots, K$, which allows us to reduce the dimensionality to $2 K$ by considering additional constraints $\boldsymbol{\delta}_{i_{k}}=\mathbf{0}, \forall k \notin \mathcal{K}$. Moreover, in this case all $\mathbf{A}_{i_{1}}, \ldots, \mathbf{A}_{i_{K}}$ are full rank matrices.

\section{A. DPCIR-based SLP Power Minimization}

In a realistic multiuser scenario, the power minimization problem might be relevant if the required QoS (e.g. SINR) of all the users can be guaranteed through the available transmission resources in the system. For a detailed discussion on the rationale behind the power minimization problem, we kindly refer the readers to [9]. Accordingly, in this section we first study the relevance of the SLP power minimization problem. For this purpose, we consider a power-restricted scenario in which the downlink transmission is supposed to provide each user with a given SINR threshold, while the BS is subject to a total power constraint. This can be interpreted as a feasibility problem based on the given power constraint and the users' SINR thresholds. Through this problem, one may examine whether the given SINR requirements are achievable or not, i.e., whether the spatial multiplexing to serve multiple users is meaningful. Otherwise, the system operator decides to relax the other constraints (e.g., decreasing the number of users, or increasing the total power budget). In the following, we first express a feasibility problem for the considered scenario and then formulate the power minimization problem.

By substituting $\mathbf{H}_{k} \tilde{\mathbf{u}}$ for $\mathbf{x}$ and scaling the distance preserving offsets $\mathbf{b}_{i_{k}}$ and $\mathbf{c}_{i_{k}}$ in (21), the CI constraint for the $k$-th user can be written as

$\mathbf{A}_{i_{k}} \mathbf{H}_{k} \tilde{\mathbf{u}}=\sigma_{k} \sqrt{\gamma_{k}}\left(\mathbf{b}_{i_{k}}+\mathbf{c}_{i_{k}}\right)+\boldsymbol{\delta}_{i_{k}}, \begin{cases}\boldsymbol{\delta}_{i_{k}} \succeq \mathbf{0} & k \in \mathcal{K}, \\ \boldsymbol{\delta}_{i_{k}}=\mathbf{0} & k \notin \mathcal{K} .\end{cases}$
Taking all $K$ users into account, (28) imposes a total number of $2 K$ constraints on the problem. Using these DPCIR constraints, the feasibility problem can be expressed as

$$
\begin{array}{cl}
\text { find } & \tilde{\mathbf{u}} \\
\text { s.t. } & \mathbf{A}_{i_{k}} \mathbf{H}_{k} \tilde{\mathbf{u}}=\sigma_{k} \sqrt{\gamma_{k}}\left(\mathbf{b}_{i_{k}}+\mathbf{c}_{i_{k}}\right)+\boldsymbol{\delta}_{i_{k}}, k=1, \ldots, K, \\
& \boldsymbol{\delta}_{i_{k}}=\mathbf{0}, \forall k \notin \mathcal{K}, \\
& \boldsymbol{\delta}_{i_{k}} \succeq \mathbf{0}, \forall k \in \mathcal{K}, \\
& \tilde{\mathbf{u}}^{\mathrm{T}} \tilde{\mathbf{u}} \leq P,
\end{array}
$$

where the forth constraint imposes the power limitation on the transmit signal. Defining $\boldsymbol{\Sigma}=\operatorname{blkdiag}\left(\sigma_{1}, \ldots, \sigma_{k}\right) \in \mathbb{R}^{2 K \times 2 K}$ and $\boldsymbol{\Gamma}=\operatorname{blkdiag}\left(\gamma_{1}, \ldots, \gamma_{k}\right) \in \mathbb{R}^{2 K \times 2 K}$, problem (29) can be written, in a more compact form, as

$$
\begin{aligned}
\text { find } & \tilde{\mathbf{u}} \\
\text { s.t. } & \mathbf{G} \tilde{\mathbf{u}}=\boldsymbol{\Sigma} \boldsymbol{\Gamma}^{1 / 2}(\mathbf{b}+\mathbf{c})+\boldsymbol{\delta}, \\
& \boldsymbol{\delta}_{i_{k}}=\mathbf{0}, \forall k \notin \mathcal{K}, \\
& \boldsymbol{\delta}_{i_{k}} \succeq \mathbf{0}, \forall k \in \mathcal{K}, \\
& \tilde{\mathbf{u}}^{\mathrm{T}} \tilde{\mathbf{u}} \leq P .
\end{aligned}
$$

A sufficient condition under which there exists (at least) a feasible point for (30) can be obtained according to the following proposition.

Proposition 1. The feasibility problem (30) has (at least) a solution for $K \leq N$ if

$$
\left\|\mathbf{G}^{\dagger} \boldsymbol{\Sigma} \boldsymbol{\Gamma}^{1 / 2}(\mathbf{b}+\mathbf{c})\right\|_{2}^{2} \leq P,
$$

where $\mathbf{G}^{\dagger}=\mathbf{G}^{\mathrm{T}}\left(\mathbf{G G}^{\mathrm{T}}\right)^{-1}$ is the Moore-Penrose inverse of G.

Proof: Let $\boldsymbol{\delta}=\mathbf{0}$, then (30) reduces to

$$
\begin{array}{cl}
\text { find } & \tilde{\mathbf{u}} \\
\text { s.t. } & \mathbf{G} \tilde{\mathbf{u}}=\boldsymbol{\Sigma} \boldsymbol{\Gamma}^{1 / 2}(\mathbf{b}+\mathbf{c}), \\
& \tilde{\mathbf{u}}^{\mathrm{T}} \tilde{\mathbf{u}} \leq P .
\end{array}
$$

Now suppose that $\tilde{\mathbf{u}}_{\mathrm{o}}=\mathbf{G}^{\dagger} \boldsymbol{\Sigma} \boldsymbol{\Gamma}^{1 / 2}(\mathbf{b}+\mathbf{c}$ ) is a solution (not necessarily unique) to the system of linear equations

$$
\mathbf{G} \tilde{\mathbf{u}}=\boldsymbol{\Sigma} \boldsymbol{\Gamma}^{1 / 2}(\mathbf{b}+\mathbf{c}) .
$$

In fact, $\tilde{\mathbf{u}}_{\mathrm{O}}$ is equal to the solution of zero-forcing beamforming (ZFBF) [33] when all the users are allocated identical SINR thresholds. We argue the existence of $\tilde{\mathbf{u}}_{o}$ as follows. In case $K=N$, due to the random channel matrices $\mathbf{H}_{k}, k=1, \ldots, K$, matrix $\mathbf{G}$ is full rank almost surely. This means that the probability of (33) having more than one solution is zero. On the other hand, for $K<N$, matrix $\mathbf{G}$ is full row rank and (33) expresses an underdetermined system of linear equations for which $\tilde{\mathbf{u}}_{\mathrm{o}}$ is the least-norm solution. Having $\tilde{\mathbf{u}}_{\mathrm{o}}$ as a solution to (33), if $\tilde{\mathbf{u}}_{\mathrm{o}}^{\mathrm{T}} \tilde{\mathbf{u}}_{\mathrm{o}} \leq P$, then $\tilde{\mathbf{u}}_{\mathrm{o}}$ is a feasible point for (32); this further ensures the feasibility of (30) since it is a relaxed version of (32). Therefore

$$
\tilde{\mathbf{u}}_{\mathrm{o}}^{\mathrm{T}} \tilde{\mathbf{u}}_{\mathrm{o}}=(\mathbf{b}+\mathbf{c})^{\mathrm{T}} \boldsymbol{\Gamma}^{1 / 2} \boldsymbol{\Sigma}\left(\mathbf{G G}^{\mathrm{T}}\right)^{\dagger} \boldsymbol{\Sigma} \boldsymbol{\Gamma}^{1 / 2}(\mathbf{b}+\mathbf{c}) \leq P,
$$

is a sufficient condition for the feasibility problem (30) to have at least one solution. 
If a solution to (30) exists, then the relevant problem is to further reduce the transmit power, which is known as power minimization. The precoder is designed to minimize either the total or the peak (per-antenna) transmit power. The latter objective is more realistic as, in practice, many systems are subject to individual per-antenna power constraints [24], [33]. Accordingly, the DPCIR-based SLP problem minimizing the total transmit power can be formulated as a quadratic programming $(\mathrm{QP})$, i.e.,

$$
\begin{aligned}
\underset{\tilde{\mathbf{u}}, \boldsymbol{\delta}}{\operatorname{minimize}} & \tilde{\mathbf{u}}^{\mathrm{T}} \tilde{\mathbf{u}} \\
\text { s.t. } & \mathbf{G} \tilde{\mathbf{u}}=\boldsymbol{\Sigma} \boldsymbol{\Gamma}^{1 / 2}(\mathbf{b}+\mathbf{c})+\boldsymbol{\delta}, \\
& \boldsymbol{\delta}_{i_{k}}=\mathbf{0}, \forall k \notin \mathcal{K}, \\
& \boldsymbol{\delta}_{i_{k}} \succeq \mathbf{0}, \forall k \in \mathcal{K},
\end{aligned}
$$

which has $2 N+2 K$ real-valued variables (included in vectors $\tilde{\mathbf{u}}$ and $\boldsymbol{\delta}$ ) and $4 K$ constraints. There are various algorithms to solve a QP optimization, e.g., interior-point or active set methods and gradient algorithms [34]. Denoting the optimal solution of (34) by $\tilde{\mathbf{u}}^{*}$, the feasibility problem (30) guarantees that $\tilde{\mathbf{u}}^{{ }^{\mathrm{T}}} \tilde{\mathbf{u}}^{*} \leq P$.

By replacing $\tilde{\mathbf{u}}^{\mathrm{T}} \tilde{\mathbf{u}}$ with $\|\tilde{\mathbf{u}}\|_{\infty, \mathbb{C}}^{2}$, the design objective aims to minimize the peak per-antenna transmit power, where by $\|\cdot\|_{\infty, \mathbb{C}}$ we mean the infinity norm over equivalent complexvalued elements. This variant of the SLP power optimization problem has convex objective function and constraints, hence is convex, and can efficiently be solved using off-the-shelf algorithms [29]. Furthermore, the feasibility problem (30) can also be extended to the case with peak per-antenna power constraints if one substitutes $\|\tilde{\mathbf{u}}\|_{\infty, \mathbb{C}}^{2}$ for $\tilde{\mathbf{u}}^{\mathrm{T}} \tilde{\mathbf{u}}$, and $P / N$ for $P$. Then, the feasibility condition is given by

$$
\left\|\mathbf{G}^{\dagger} \boldsymbol{\Sigma} \boldsymbol{\Gamma}^{1 / 2}(\mathbf{b}+\mathbf{c})\right\|_{\infty, \mathbb{C}}^{2} \leq P / N
$$

It is worth noting that if the condition in (35) holds, then the feasibility condition in Proposition 1 is also satisfied given the norm inequality

$$
\left\|\mathbf{G}^{\dagger} \boldsymbol{\Sigma} \boldsymbol{\Gamma}^{1 / 2}(\mathbf{b}+\mathbf{c})\right\|_{2} \leq \sqrt{N}\left\|\mathbf{G}^{\dagger} \boldsymbol{\Sigma} \boldsymbol{\Gamma}^{1 / 2}(\mathbf{b}+\mathbf{c})\right\|_{\infty, \mathbb{C}} .
$$

It is possible to further simplify the power minimization problem (in terms of problem size) by reducing the number of optimization variables and constraints as below.

Lemma 4. The $Q P$ in (34) can be reduced to

$$
\underset{\boldsymbol{\delta} \succeq \mathbf{0}}{\operatorname{minimize}}\left\|\mathbf{G}^{\dagger}\left(\boldsymbol{\Sigma} \boldsymbol{\Gamma}^{1 / 2}(\mathbf{b}+\mathbf{c})+\mathbf{W} \boldsymbol{\delta}\right)\right\|_{2}^{2},
$$

for $K \leq N$, where $\mathbf{W}$ is an $2 K \times 2 K$ diagonal matrix with a diagonal element being one if it corresponds to a user in $\mathcal{K}$ and zero otherwise. The optimal transmit vector $\tilde{\mathbf{u}}^{*}$ is then obtained by

$$
\tilde{\mathbf{u}}^{*}=\mathbf{G}^{\dagger}\left(\boldsymbol{\Sigma} \boldsymbol{\Gamma}^{1 / 2}(\mathbf{b}+\mathbf{c})+\mathbf{W} \boldsymbol{\delta}^{*}\right),
$$

where $\delta^{*}$ is the optimum of (36).

Proof: To verify the equivalence of problems (34) and (36), let consider two cases. If $K=N$, then $\mathbf{G}$ is almost surely full rank, and hence $\mathbf{G}^{\dagger}=\mathbf{G}^{-1}$. As a result, the constraint $\mathbf{G} \tilde{\mathbf{u}}=\boldsymbol{\Sigma} \boldsymbol{\Gamma}^{1 / 2}(\mathbf{b}+\mathbf{c})+\boldsymbol{\delta}$ in (34) gives a unique solution for any fixed $\delta$. In such case, there would be a bijection between $\boldsymbol{\delta}$ and $\tilde{\mathbf{u}}$, which implies that one of them can be obtained as a one-to-one function of the other. Therefore, optimizing $\delta$ is equivalent to optimizing both $\tilde{\mathbf{u}}$ and $\boldsymbol{\delta}$. Otherwise if $K<N$, the constraint $\mathbf{G} \tilde{\mathbf{u}}=\boldsymbol{\Sigma} \boldsymbol{\Gamma}^{1 / 2}(\mathbf{b}+\mathbf{c})+\boldsymbol{\delta}$ may have more than one solution (since $\mathbf{G}$ is full row rank in this case). Therefore, the QP in (34) can be written as

$$
\begin{array}{ccl}
\underset{\boldsymbol{\delta}}{\operatorname{minimize}} & \min _{\tilde{\mathbf{u}}} & \tilde{\mathbf{u}}^{\mathrm{T}} \tilde{\mathbf{u}} \\
& \text { s.t. } & \mathbf{G} \tilde{\mathbf{u}}=\boldsymbol{\Sigma} \boldsymbol{\Gamma}^{1 / 2}(\mathbf{b}+\mathbf{c})+\boldsymbol{\delta}, \\
& \boldsymbol{\delta}_{i_{k}}=\mathbf{0}, \forall k \notin \mathcal{K}, \\
& \boldsymbol{\delta}_{i_{k}} \succeq \mathbf{0}, \forall k \in \mathcal{K},
\end{array}
$$

in which the solution of the inner minimization is given by $\tilde{\mathbf{u}}=\mathbf{G}^{\dagger}\left(\boldsymbol{\Sigma} \boldsymbol{\Gamma}^{1 / 2}(\mathbf{b}+\mathbf{c})+\boldsymbol{\delta}\right)$, i.e., the least-norm solution to the system of linear equations $\mathbf{G} \tilde{\mathbf{u}}=\boldsymbol{\Sigma} \boldsymbol{\Gamma}^{1 / 2}(\mathbf{b}+\mathbf{c})+\boldsymbol{\delta}$. In addition, the diagonal matrix $\mathbf{W}$ imposes $\boldsymbol{\delta}_{i_{k}}=\mathbf{0}$ for all $k \notin \mathcal{K}$, if any exist.

The reduced QP in (36) involves $2 K$ variables and $2 K$ constraints, hence is less computationally complex than the original QP in (34) due to a smaller problem size. Moreover, problem (36) can be classified as a non-negative least squares (NNLS) optimization and solved using known efficient methods, e.g., fast NNLS algorithm [35].

\section{B. DPCIR-based SLP SINR Balancing}

In a downlink scenario where power is a strict transmit limitation, fairness might be a relevant design criterion [6]. In this paper, we are interested in max-min fairness criterion under which the SLP design problem aims at maximizing the worst SINR among all users, limited by a total transmit power $P$. Assuming the CIRs to be distance preserving, the problem is not convex in its original form. In this section, we first provide an overview and discuss the methods presented in the literature to solve the SLP max-min SINR problem. Then, we derive several alternate convex formulations for this problem. All the proposed methods are simulated in Section $\mathrm{V}$ with a detailed discussion on complexity and performance.

One may tackle the SLP max-min SINR by exploiting its connection to the power minimization, as proposed in [18]. By considering the DPCIR-based design as a generalization of [18], this method iteratively solves

$$
\begin{aligned}
\tilde{\mathbf{u}}_{\mathrm{PM}}\left(\boldsymbol{\Gamma}^{*}\right)=\underset{\tilde{\mathbf{u}}, \boldsymbol{\delta}}{\operatorname{argmin}} & \tilde{\mathbf{u}}^{\mathrm{T}} \tilde{\mathbf{u}} \\
\text { s.t. } & \mathbf{G} \tilde{\mathbf{u}}=\boldsymbol{\Sigma} \boldsymbol{\Gamma}^{*^{1 / 2}}(\mathbf{b}+\mathbf{c})+\boldsymbol{\delta}, \\
& \boldsymbol{\delta}_{i_{k}}=\mathbf{0}, \forall k \notin \mathcal{K}, \\
& \boldsymbol{\delta}_{i_{k}} \succeq \mathbf{0}, \forall k \in \mathcal{K},
\end{aligned}
$$

where $\boldsymbol{\Gamma}^{*}=\operatorname{blkdiag}\left(\gamma_{1}^{*}, \ldots, \gamma_{K}^{*}\right)$ is the input vector of SINR thresholds given by the optimal solution of

$$
\begin{aligned}
\tilde{\mathbf{u}}_{\mathrm{SB}}(P)=\underset{\tilde{\mathbf{u}}, \boldsymbol{\Gamma}, \boldsymbol{\delta}}{\arg \max } & \min _{k}\left\{\gamma_{k}\right\}_{k=1}^{K} \\
\text { s.t. } & \mathbf{G} \tilde{\mathbf{u}}=\boldsymbol{\Sigma} \boldsymbol{\Gamma}^{1 / 2}(\mathbf{b}+\mathbf{c})+\boldsymbol{\delta}, \\
& \boldsymbol{\delta}_{i_{k}}=\mathbf{0}, \forall k \notin \mathcal{K}, \\
& \boldsymbol{\delta}_{i_{k}} \succeq \mathbf{0}, \forall k \in \mathcal{K}, \\
& \tilde{\mathbf{u}}^{\mathrm{T}} \tilde{\mathbf{u}} \leq P,
\end{aligned}
$$


until the (minimum power) solution of (39) converges to $P$. It can be inferred that the power optimization (39) and the max-min SINR (40) are related as

$$
\tilde{\mathbf{u}}_{\mathrm{PM}}\left(\boldsymbol{\Gamma}^{*}\right)=\tilde{\mathbf{u}}_{\mathrm{SB}}\left(\tilde{\mathbf{u}}_{\mathrm{PM}}\left(\boldsymbol{\Gamma}^{*}\right)^{\mathrm{T}} \tilde{\mathbf{u}}_{\mathrm{PM}}\left(\boldsymbol{\Gamma}^{*}\right)\right) .
$$

In fact, $\gamma_{k}$ in (40) manipulates the instantaneous average power of the constellation, from which $\mathcal{D}_{i_{k} \text {,DP }}$ is constructed, in order to ensure $\tilde{\mathbf{u}}^{\mathrm{T}} \mathbf{H}_{k}^{\mathrm{T}} \mathbf{H}_{k} \tilde{\mathbf{u}} \geq \sigma_{k}^{2} \gamma_{k}$ through the first constraint. This is a conservative way to guarantee that the instantaneous SINR satisfies $\mathbb{E}_{\mathrm{t}}\left\{\tilde{\mathbf{u}}^{\mathrm{T}} \mathbf{H}_{k}^{\mathrm{T}} \mathbf{H}_{k} \tilde{\mathbf{u}}\right\} / \sigma_{k}^{2} \geq \gamma_{k}, k=$ $1, \ldots, K$, which is typically desired in conventional multiuser precoding [4]. The optimal solution $\gamma_{k}^{*}$, however, pushes $\mathbf{H}_{k} \tilde{\mathbf{u}}$ into $\sqrt{\gamma_{k}^{*}} \mathcal{D}_{i_{k}, \text { DP }}$ rather than $\mathcal{D}_{i_{k}, \mathrm{DP}}$. Since $\gamma_{k}^{*}$ is a function of the users' symbol vector $\mathbf{s}$, it varies over symbol time, limiting the applicability of this method to constant envelope modulations. For generic constellations, possibly having interior points (with bounded decision regions), the $k$-th receiver needs to be aware of the value of $\gamma_{k}^{*}$ in each symbol period in order to correctly detect $s_{k}$, which is practically unrealistic. It is important to note that we are not allowed to reformulate (40) by excluding the constraints related to the users $k \notin \mathcal{K}$, as the power optimization (39) needs to take all the users' symbols into account in order to guarantee the given SINR thresholds for all $K$ users.

Assuming identical noise distributions across the receivers, i.e., $\sigma_{k}^{2}=\sigma^{2}, k=1, \ldots, K$, the symbol-level SINR for user $k$ is proportional to the instantaneous received power at the $k$-th receiver in each symbol time. On this account, the DPCIRbased SLP max-min SINR problem can be formulated as

$$
\begin{aligned}
\underset{\tilde{\mathbf{u}}, \boldsymbol{\delta}}{\operatorname{maximize}} & \min _{k}\left\{\tilde{\mathbf{u}}^{\mathrm{T}} \mathbf{H}_{k}^{\mathrm{T}} \mathbf{H}_{k} \tilde{\mathbf{u}}\right\}_{k \in \mathcal{K}} \\
\text { s.t. } & \mathbf{G} \tilde{\mathbf{u}}=\boldsymbol{\Sigma}(\mathbf{b}+\mathbf{c})+\boldsymbol{\delta}, \\
& \boldsymbol{\delta}_{i_{k}}=\mathbf{0}, \forall k \notin \mathcal{K}, \\
& \boldsymbol{\delta}_{i_{k}} \succeq \mathbf{0}, \forall k \in \mathcal{K}, \\
& \tilde{\mathbf{u}}^{\mathrm{T}} \tilde{\mathbf{u}} \leq P .
\end{aligned}
$$

By introducing a slack variable $\lambda$, one can recast (42) as

$$
\begin{aligned}
\underset{\tilde{\mathbf{u}}, \boldsymbol{\delta}, \lambda \geq 0}{\operatorname{maximize}} & \lambda \\
\text { s.t. } & \mathbf{G} \tilde{\mathbf{u}}=\mathbf{\Sigma}(\mathbf{b}+\mathbf{c})+\boldsymbol{\delta}, \\
& \tilde{\mathbf{u}}^{\mathrm{T}} \mathbf{H}_{k}^{\mathrm{T}} \mathbf{H}_{k} \tilde{\mathbf{u}} \geq \lambda, k \in \mathcal{K}, \\
& \boldsymbol{\delta}_{i_{k}}=\mathbf{0}, \forall k \notin \mathcal{K}, \\
& \boldsymbol{\delta}_{i_{k}} \succeq \mathbf{0}, \forall k \in \mathcal{K}, \\
& \tilde{\mathbf{u}}^{\mathrm{T}} \tilde{\mathbf{u}} \leq P,
\end{aligned}
$$

which is not convex due to the second set of constraints. In order to deal with this problem, we use the properties of DPCIRs derived in Section III. According to Remark 2, any point in $\mathcal{D}_{i_{k}, \text { DP }}$ can be uniquely specified by $\boldsymbol{\delta}_{i_{k}}=\left[\delta_{i_{k}, 1}, \delta_{i_{k}, 2}\right]^{\mathrm{T}} \in \mathbb{R}_{+}^{2}$ for all $\mathbf{x}_{i_{k}} \in \mathbf{b d} \mathcal{X}$. It then follows from Theorem 1 that $\tilde{\mathbf{u}}^{\mathrm{T}} \mathbf{H}_{k}^{\mathrm{T}} \mathbf{H}_{k} \tilde{\mathbf{u}}=\left\|\mathbf{H}_{k} \tilde{\mathbf{u}}\right\|_{2}^{2}$ is strictly increasing in each element of $\boldsymbol{\delta}_{i_{k}}$ for all $k \in \mathcal{K}$, i.e., letting either $\delta_{i_{k}, 1}$ or $\delta_{i_{k}, 2}$ be fixed, $\tilde{\mathbf{u}}^{\mathrm{T}} \mathbf{H}_{k}^{\mathrm{T}} \mathbf{H}_{k} \tilde{\mathbf{u}}$ is a monotonically increasing function of the other. This suggests that in case the optimal value of one of the elements, e.g., $\delta_{i_{k}, 1}$, is given for any user $k \in \mathcal{K}$, then maximizing $\tilde{\mathbf{u}}^{\mathrm{T}} \mathbf{H}_{k}^{\mathrm{T}} \mathbf{H}_{k} \tilde{\mathbf{u}}$ is equivalent to maximizing $\delta_{i_{k}, 2}$. In other words, by fixing one of the variables $\delta_{i_{k}, 1}$ or $\delta_{i_{k}, 2}$ for all users $k \in \mathcal{K}$, the problem can be formulated as a convex optimization problem. Let assume $\delta_{i_{k}, 1}, \forall k \in \mathcal{K}$ are fixed, then the convex reformulation of (43) can be written as

$$
\begin{aligned}
\underset{\tilde{\mathbf{u}}, \boldsymbol{\delta} \backslash \boldsymbol{\delta}_{\mathcal{K}, 1}, \lambda \geq 0}{\operatorname{maximize}} & \lambda \\
\text { s.t. } & \mathbf{G} \tilde{\mathbf{u}}=\boldsymbol{\Sigma}(\mathbf{b}+\mathbf{c})+\boldsymbol{\delta}, \\
& \boldsymbol{\delta}_{i_{k}}=\mathbf{0}, \forall k \notin \mathcal{K}, \\
& \delta_{i_{k}, 2} \geq \lambda, \forall k \in \mathcal{K}, \\
& \tilde{\mathbf{u}}^{\mathrm{T}} \tilde{\mathbf{u}} \leq P,
\end{aligned}
$$

where $\delta_{i_{k}, 2}$ is substituted for $\tilde{\mathbf{u}}^{\mathrm{T}} \mathbf{H}_{k}^{\mathrm{T}} \mathbf{H}_{k} \tilde{\mathbf{u}}$ in (43), and $\boldsymbol{\delta}_{\mathcal{K}, 1} \in$ $\mathbb{R}_{+}^{|\mathcal{K}|}$ denotes the vector collecting $\delta_{i_{k}, 1}$ for all $k \in \mathcal{K}$.

In theory, achieving the optimum of (43) through (44) requires a complete search over all possible (non-negative) values of $\delta_{i_{k}, 1}, k \in \mathcal{K}$, solving (44) for each choice, and finally picking the maximum among all the candidate solutions. Due to the power limitation induced by $P$, one may bound and discretize the search interval to do a grid search. This reduces the optimization to choose $\delta_{i_{k}, 1}, k \in \mathcal{K}$ from a finite set, but of course leads to a sub-optimal solution. Considering an identical search interval for all users, let $N_{\delta}$ be the number of discrete values for $\delta_{i_{k}, 1}, k \in \mathcal{K}$, which results in a total number of $N_{\delta}^{|\mathcal{K}|}$ combinations over all $|\mathcal{K}|$ users. This means that the number of convex problems to be solved every symbol time is of order $N_{\delta}^{|\mathcal{K}|}$. In general, the gap to the optimal solution depends on $N_{\delta}$, as well as the bounding accuracy (i.e., whether the search interval includes the optimal value or not). The output of the grid search tends to the optimum of (43) as $N_{\delta} \rightarrow \infty$, however, the computational complexity grows exponentially with $N_{\delta}$. Motivated by the very high and impractical complexity of the grid search method, we propose two more computationally tractable approaches to solve the SLP max-min SINR problem. The proposed alternatives are not equivalent to the original problem (43), but extensively reduce the computational complexity of the solution method compared to the grid search. In Section V, the loss due to each proposed alternative will be evaluated through simulation results.

1) Semidefinite programming formulation: Inspired by the increasing monotonicity of $\tilde{\mathbf{u}}^{\mathrm{T}} \mathbf{H}_{k}^{\mathrm{T}} \mathbf{H}_{k} \tilde{\mathbf{u}}$ with respect to both elements of $\boldsymbol{\delta}_{i_{k}}$ for all $k \in \mathcal{K}$, we propose an alternative way to convert (43) into a convex problem by replacing the non-convex quadratic constraints on $\tilde{\mathbf{u}}^{\mathrm{T}} \mathbf{H}_{k}^{\mathrm{T}} \mathbf{H}_{k} \tilde{\mathbf{u}}$ with affine constraints on $\boldsymbol{\delta}_{i_{k}}$, i.e.,

$$
\begin{aligned}
\underset{\tilde{\mathbf{u}}, \boldsymbol{\delta}, \lambda \geq 0}{\operatorname{maximize}} & \lambda \\
\text { s.t. } & \mathbf{G} \tilde{\mathbf{u}}=\boldsymbol{\Sigma}(\mathbf{b}+\mathbf{c})+\boldsymbol{\delta}, \\
& \boldsymbol{\delta}_{i_{k}}=\mathbf{0}, \forall k \notin \mathcal{K}, \\
& \boldsymbol{\delta}_{i_{k}} \succeq \lambda \mathbf{1}, k \in \mathcal{K}, \\
& \tilde{\mathbf{u}}^{\mathrm{T}} \tilde{\mathbf{u}} \leq P
\end{aligned}
$$

which can be interpreted as jointly maximizing $\delta_{i_{k}, 1}$ and $\delta_{i_{k}, 2}$ over all $k \in \mathcal{K}$. By Schur complement, problem (45) can be 
written as

$$
\begin{aligned}
\underset{\tilde{\mathbf{u}}, \boldsymbol{\delta}, \lambda \geq 0}{\operatorname{maximize}} & \lambda \\
\text { s.t. } & \mathbf{G} \tilde{\mathbf{u}}=\boldsymbol{\Sigma}(\mathbf{b}+\mathbf{c})+\boldsymbol{\delta}, \\
& \boldsymbol{\delta}_{i_{k}}=\mathbf{0}, \forall k \notin \mathcal{K}, \\
& {\left[\begin{array}{cc}
\operatorname{diag}\left(\boldsymbol{\delta}_{\mathcal{K}}\right) & \mathbf{I}_{2|\mathcal{K}|} \\
\mathbf{I}_{2|\mathcal{K}|} & \lambda \mathbf{I}_{2|\mathcal{K}|}
\end{array}\right] \succeq 0, } \\
& {\left[\begin{array}{cc}
1 & \tilde{\mathbf{u}}^{\mathrm{T}} \\
\tilde{\mathbf{u}} & P \mathbf{I}_{2 N}
\end{array}\right] \succeq 0, }
\end{aligned}
$$

where $\boldsymbol{\delta}_{\mathcal{K}} \in \mathbb{R}_{+}^{2|\mathcal{K}|}$ is the vector collecting $\boldsymbol{\delta}_{i_{k}}$ for all $k \in$ $\mathcal{K}$, and $\succeq 0$ denotes positive semidefinite. Problem (46) is a semidefinite programming (SDP) and can be solved using known algorithms [29]. This convex formulation, however, is not expected to achieve the same solution as compared to the original problem (43) since it has a reduced degrees of freedom to maximize the minimum SINR. More precisely, the SDP in (46) optimizes $\min \left\{\delta_{i_{k}, 1}, \delta_{i_{k}, 2}\right\}$ instead of optimizing both $\delta_{i_{k}, 1}$ and $\delta_{i_{k}, 2}$. Nonetheless, the optimal solution of problem (46) can be regarded as a lower bound on the optimum of the SLP max-min SINR. It is also important to note that the SDP (46) is equivalent to the SOCP formulation of SLP SINR balancing proposed for PSK constellations in [17]. However, the SOCP formulation in [17] is not equivalent to the original SLP max-min SINR problem.

2) Block Coordinate Descent Optimization: In order to improve the solution of the SDP formulation (46), we propose an iterative method based on the block coordinate descent (BCD) algorithm [36]. The BCD is a family of successive lowerbound maximization methods in which certain approximate version of the objective function is optimized with respect to one block variable at a time, while fixing the rest of the block variables. We denote by $\boldsymbol{\delta}_{\mathcal{K}, 1} \in \mathbb{R}_{+}^{|\mathcal{K}|}$ and $\boldsymbol{\delta}_{\mathcal{K}, 2} \in \mathbb{R}_{+}^{|\mathcal{K}|}$ the vectors (blocks) collecting $\delta_{i_{k}, 1}$ and $\delta_{i_{k}, 2}$ for all $k \in \mathcal{K}$, respectively. The idea behind the BCD-based method is then to successively maximize the worst-user SINR along coordinates $\boldsymbol{\delta}_{\mathcal{K}, 1}$ and $\boldsymbol{\delta}_{\mathcal{K}, 2}$ until convergence is reached. In more detail, by defining the (elementwise) monotonically increasing function $f_{k}: \mathbb{R}_{+}^{2} \mapsto \mathbb{R}$ as

$$
f_{k}\left(\delta_{i_{k}, 1}, \delta_{i_{k}, 2}\right)=\tilde{\mathbf{u}}^{\mathrm{T}} \mathbf{H}_{k}^{\mathrm{T}} \mathbf{H}_{k} \tilde{\mathbf{u}}, k \in \mathcal{K},
$$

the objective function of the SLP max-min SINR can be expressed as

$$
g\left(\boldsymbol{\delta}_{\mathcal{K}, 1}, \boldsymbol{\delta}_{\mathcal{K}, 2}\right)=\min _{k}\left\{f_{k}\left(\delta_{i_{k}, 1}, \delta_{i_{k}, 2}\right)\right\}_{k \in \mathcal{K}} .
$$

At iteration $n$, each block of variables is updated by using the following objective functions (the constraints are as before):

$$
\begin{gathered}
\boldsymbol{\delta}_{\mathcal{K}, 1 \mid n}^{*}=\underset{\boldsymbol{\delta}_{\mathcal{K}, 1}}{\operatorname{argmax}} \quad g\left(\boldsymbol{\delta}_{\mathcal{K}, 1}, \boldsymbol{\delta}_{\mathcal{K}, 2 \mid n-1}^{*}\right), \\
\boldsymbol{\delta}_{\mathcal{K}, 2 \mid n}^{*}=\underset{\boldsymbol{\delta}_{\mathcal{K}, 2}}{\operatorname{argmax}} \quad g\left(\boldsymbol{\delta}_{\mathcal{K}, 1 \mid n-1}^{*}, \boldsymbol{\delta}_{\mathcal{K}, 2}\right),
\end{gathered}
$$

where $\boldsymbol{\delta}_{\mathcal{K}, 1 \mid n}^{*}$ and $\boldsymbol{\delta}_{\mathcal{K}, 2 \mid n}^{*}$ respectively denote the optimal solutions of (49) and (50) obtained at the $n$-th iteration, and $g\left(\boldsymbol{\delta}_{\mathcal{K}, 1}, \boldsymbol{\delta}_{\mathcal{K}, 2 \mid n-1}^{*}\right)$ and $g\left(\boldsymbol{\delta}_{\mathcal{K}, 1 \mid n-1}^{*}, \boldsymbol{\delta}_{\mathcal{K}, 2}\right)$ are lower approximations of $g\left(\boldsymbol{\delta}_{\mathcal{K}, 1}, \boldsymbol{\delta}_{\mathcal{K}, 2}\right)$. We adopt a cyclic updating rule, i.e.,

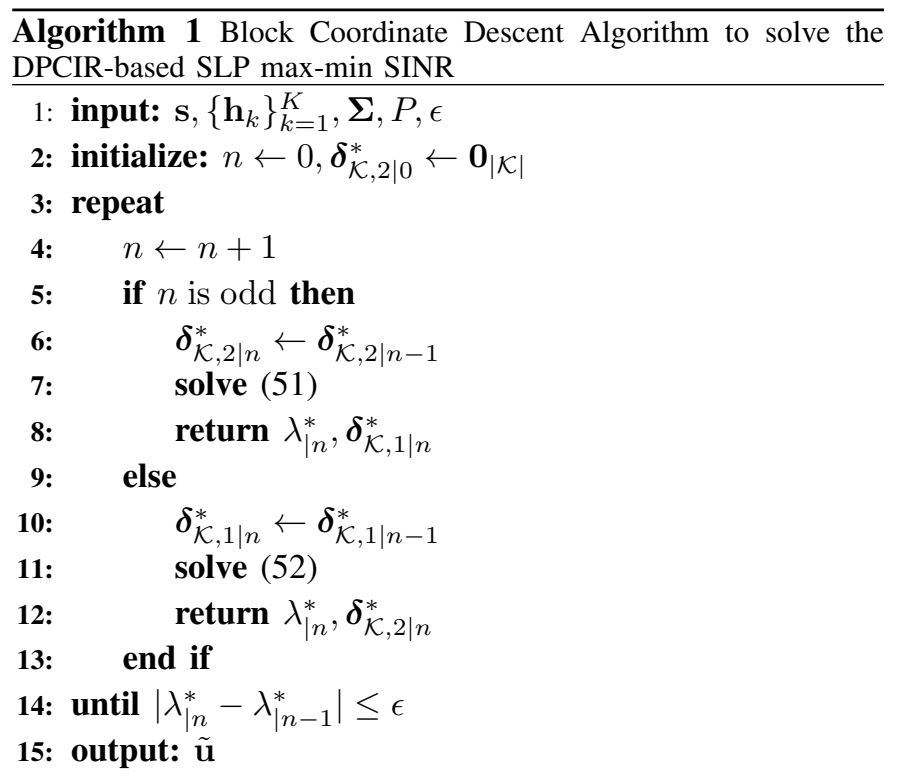

the BCD algorithm cyclically solves the following two SDPs:

$$
\begin{aligned}
\underset{\tilde{\mathbf{u}}, \boldsymbol{\delta}_{\mathcal{K}, 1}, \lambda \geq 0}{\operatorname{maximize}} & \lambda \\
\text { s.t. } & \mathbf{G} \tilde{\mathbf{u}}=\boldsymbol{\Sigma}(\mathbf{b}+\mathbf{c})+\boldsymbol{\delta}, \\
& \boldsymbol{\delta}_{i_{k}}=\mathbf{0}, \forall k \notin \mathcal{K}, \\
& {\left[\begin{array}{cc}
\operatorname{diag}\left(\boldsymbol{\delta}_{\mathcal{K}, 1}\right) & \mathbf{I} \\
\mathbf{I} & \lambda \mathbf{I}
\end{array}\right] \succeq 0, } \\
& {\left[\begin{array}{cc}
1 & \tilde{\mathbf{u}}^{\mathrm{T}} \\
\tilde{\mathbf{u}} & P \mathbf{I}
\end{array}\right] \succeq 0, }
\end{aligned}
$$

and

$$
\begin{aligned}
\underset{\tilde{\mathbf{u}}, \boldsymbol{\delta}_{\mathcal{K}, 2, \lambda \geq 0}}{\operatorname{maximize}} & \lambda \\
\text { s.t. } & \mathbf{G} \tilde{\mathbf{u}}=\boldsymbol{\Sigma}(\mathbf{b}+\mathbf{c})+\boldsymbol{\delta}, \\
& \boldsymbol{\delta}_{i_{k}}=\mathbf{0}, \forall k \notin \mathcal{K}, \\
& {\left[\begin{array}{cc}
\operatorname{diag}\left(\boldsymbol{\delta}_{\mathcal{K}, 2}\right) & \mathbf{I} \\
\mathbf{I} & \lambda \mathbf{I}
\end{array}\right] \succeq 0, } \\
& {\left[\begin{array}{cc}
1 & \tilde{\mathbf{u}}^{\mathrm{T}} \\
\tilde{\mathbf{u}} & P \mathbf{I}
\end{array}\right] \succeq 0, }
\end{aligned}
$$

where the dimensions of identity matrices in (51) and (52) are the same as in (46). Each SDP is solved with respect to one of the blocks $\boldsymbol{\delta}_{\mathcal{K}, 1}$ or $\boldsymbol{\delta}_{\mathcal{K}, 2}$ while the other block is fixed and is given by the solution of the previous iteration. The pseudocode of the proposed method is presented in Algorithm 1, where we have arbitrarily initialized $\boldsymbol{\delta}_{\mathcal{K}, 2}^{*}$. For all iterations $n=1,2, \ldots$, we have

$$
\boldsymbol{\delta}_{\mathcal{K}, 1 \mid n-1}^{*} \preceq \boldsymbol{\delta}_{\mathcal{K}, 1 \mid n}^{*}, \quad \boldsymbol{\delta}_{\mathcal{K}, 2 \mid n-1}^{*} \preceq \boldsymbol{\delta}_{\mathcal{K}, 2 \mid n}^{*},
$$

and hence

$$
\lambda_{\mid n-1}^{*} \leq \lambda_{\mid n}^{*},
$$

where by $\lambda_{\mid n}^{*}$ we denote the optimal solution at the $n$-th iteration. The sequence $\left\{\lambda_{\mid n}^{*}\right\}_{n=1,2, \ldots}$ is therefore guaranteed to converge to a stationary point (local optimum) of the SLP max-min SINR. As we will see in Section V, the BCD algorithm usually converges after a few iterations. 


\section{Simulation Results}

In this section, we provide simulation results to validate the analytical discussion in earlier sections and also to evaluate the performance of the proposed SLP design approaches. We also compare our results with the state of the art. In all the simulations, we consider a downlink multiuser unicast scenario (with equal number of transmit and receive antennas, i.e., $N=K$ ) in which intended symbols for all the users are taken from an identical constellation set. We evaluate the results for three constellations, namely, 8-PSK, optimized 8-ary and 16QAM; however, we are particularly interested in the optimized 8 -ary constellation since it has a generic shape with unequal distances as well as both bounded and unbounded Voronoi regions. We assume the variance of the noise component at the receiver of each user to be $\sigma_{k}^{2}=\sigma^{2}=1, k=1, \ldots, K$. Furthermore, we assume equal SINR thresholds $\gamma_{k}=\gamma, k=$ $1, \ldots, K$ when the power minimization is of interest. A quasistatic Rayleigh fading channel is assumed where the complex channel vector $\mathbf{h}_{k}, k=1, \ldots, K$ is generated following an i.i.d. complex Gaussian distribution with zero-mean and unit variance, with assumption $\mathbb{E}\left\{\mathbf{h}_{k} \mathbf{h}_{j}^{\mathrm{H}}\right\}=0, \forall j=1, \ldots, K, j \neq k$. As for the BCD algorithm, we set the terminating condition $\epsilon=10^{-3}$ with a maximum number of iterations 100 .

For a power-limited downlink scenario with $N=K=4$, the feasibility probability of the DPCIR-based SLP scheme is obtained (based on Proposition 1) and shown in Fig. 3. The adopted constellation is the optimized 8-ary, and the probabilities are calculated by averaging over all $8^{4}$ possible combinations of the users' symbol vector $\mathbf{s}$, and further averaging over 1000 randomly generated channel realizations. It can be noticed that for smaller values of $\gamma$, the probability of feasibility grows faster as a function of the total transmit power budget. A case-specific example could be wireless systems with adaptive coding and modulation (ACM) capability, such as DVB-S2X broadcasting standard [37]. In DVB-S2X, the target range of SNR for an 8-ary constellation is typically around 5-7 dB over a linear channel (recall that in SLP, SINR can be interpreted as the received SNR). In such system with a total power budget of at least $130 \mathrm{dBW}$, one can infer from Fig. 3 that providing all the users with an SINR (SNR) level of $\gamma=5 \mathrm{~dB}$ is guaranteed by $90 \%$, and further reduction of transmit power might be possible via the SLP power optimization.

In Fig. 4, we plot the average achievable throughput of $K=8$ users under the SLP power minimization scheme as a function of a given target rate $R$, where the target rate is related to the SINR threshold by $R=\log _{2}(1+\gamma)$. The number of BS's transmit antennas is $N=8$ and 8-PSK constellation is employed. The average achievable throughput for the $k$-th user is defined as

$$
\left(1-\mathrm{SER}_{k}\right) \log _{2}\left(1+\mathbb{E}_{\mathrm{t}}\left\{\left\|\mathbf{h}_{k} \mathbf{u}\right\|_{2}^{2}\right\}\right),
$$

where $\mathrm{SER}_{k}$ is the symbol error rate of user $k$, and the expectation is taken over the whole frame. In addition to the DPCIR-based SLP design, the results are obtained for two other SLP approaches, namely, constructive interference zero-forcing (CIZF) and constructive interference power minimization (CIPM) [18]. The proposed DPCIR-based scheme

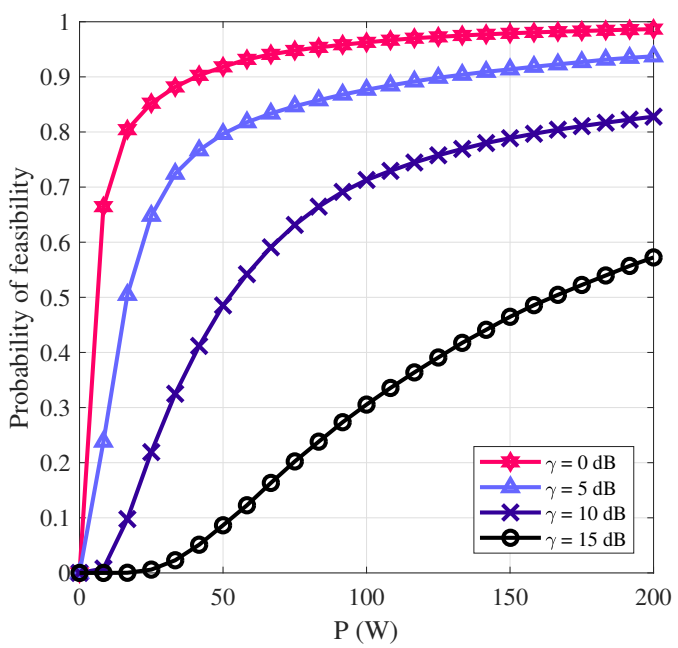

Fig. 3. Feasibility probability of SLP as a function of the transmit power budget for different SINR thresholds with $N=K=4$.

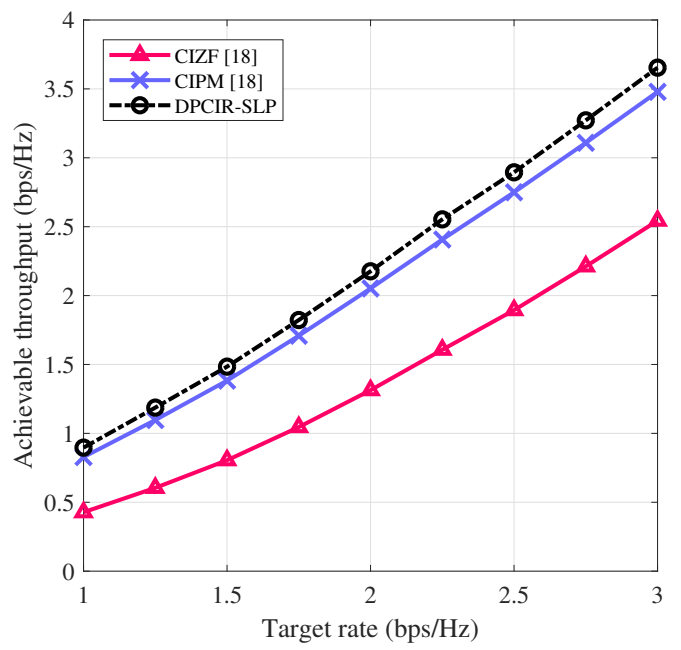

Fig. 4. Average per-user achievable throughput as a function of target rate with $N=K=8$.

outperforms both CIZF and CIPM. It can also be observed that both the DPCIR-based and the CIPM symbol-level precoders provide higher achievable throughputs than the given target rate. Moreover, under same scenario, the average symbol error probability over all $K$ users is depicted versus SINR threshold in Fig. 5. As it can be seen, defining the CIR constraints of the SLP power optimization to be distance preserving causes a very slight difference in the average SER compared to the CIPM approach (in which the phase of the noise-free received signal is pushed to agree with that of the original constellation point). Overall, with respect to Fig. 4, the DPCIR-based SLP shows a better performance than the CIPM in terms of the achievable throughput given by (55) which takes into account both the shape of the CIR and the resulting SER.

Figure 6 shows the scatter plot of $K \times 10^{3}$ noise-free received signals in a scenario with $K=N=8$ and $\gamma=15$ $\mathrm{dBW}$, where all the transmitted symbols are drawn from 8PSK constellation and mapped to $N$ transmit antennas via 


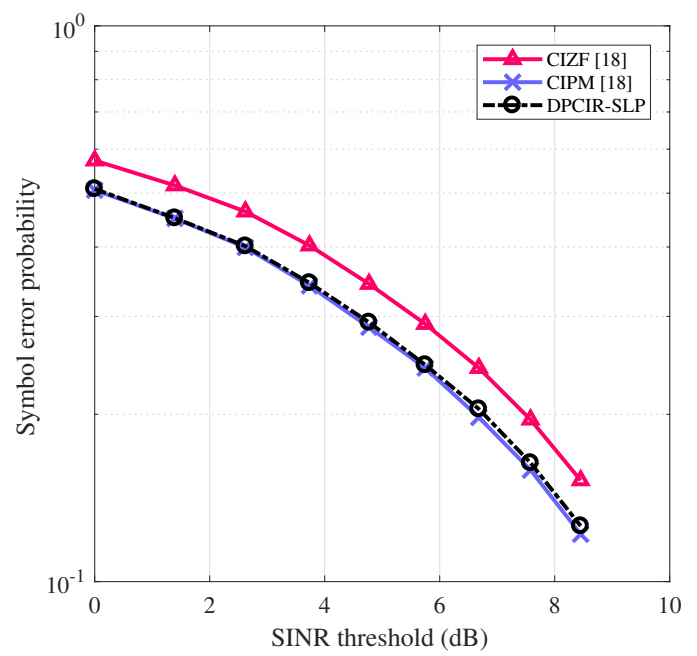

Fig. 5. Average symbol error probability as a function of SINR threshold with $N=K=8$.

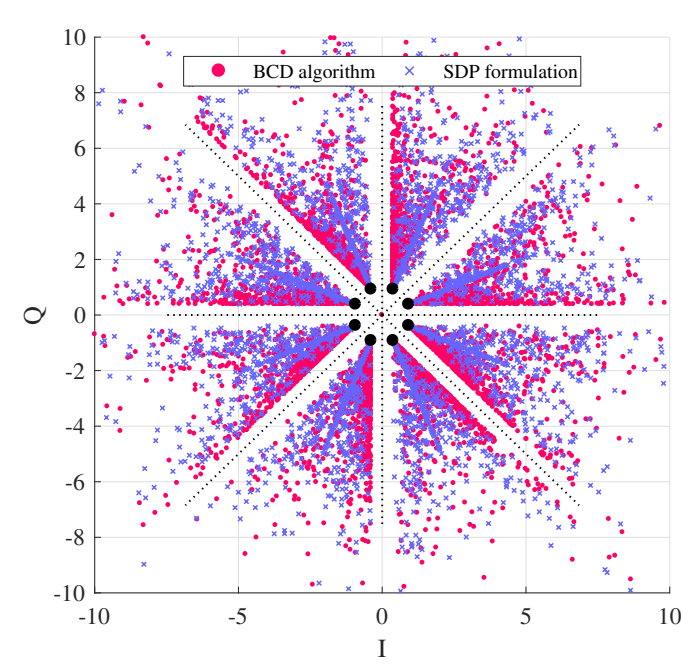

Fig. 6. Scatter plot of the noise-free received signals taken form 8-PSK constellation with $N=K=8$ and $\gamma=15 \mathrm{dBW}$. The black points and the dashed lines represent the constellation points and their corresponding Voronoi regions, respectively.

a DPCIR-based SLP max-min SINR precoder. This figure confirms the discussion in Section IV regarding the relative geometry of the noise-free received signal in a DPCIR. It can be seen from Fig. 6 that the density of signals resulted from the $\mathrm{BCD}$ algorithm is higher in areas closer to the boundaries of DPCIRs, while those signals from the SDP formulation are distributed around the bisector (with the majority located exactly on the bisector). This is a consequence of maximizing the minimum of $\delta_{i_{k}, 1}$ and $\delta_{i_{k}, 2}$ in (46) which, loosely speaking, disregards half of the degrees of freedom in optimization. On the other hand, as it can be seen in Fig. 6, the results obtained from the BCD algorithm are biased towards one of the boundaries in each DPCIR, depending on the initialization step (i.e., whether to initialize $\boldsymbol{\delta}_{\mathcal{K}, 1}$ or $\boldsymbol{\delta}_{\mathcal{K}, 2}$ ). Exactly same trend as in Fig. 6 can be obtained for the output of the SOCP formulation of SLP max-min SINR in [17].

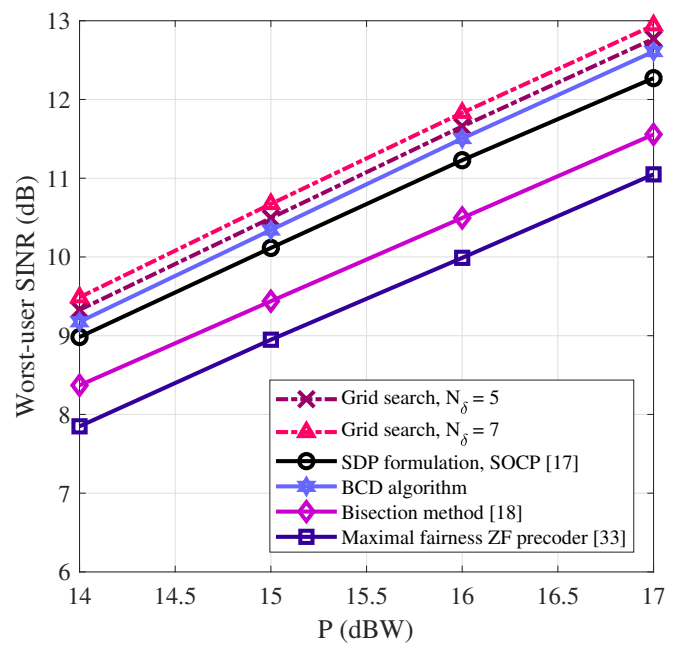

Fig. 7. Minimum SINR among $K=4$ users versus total power constraint for 8-PSK constellation.

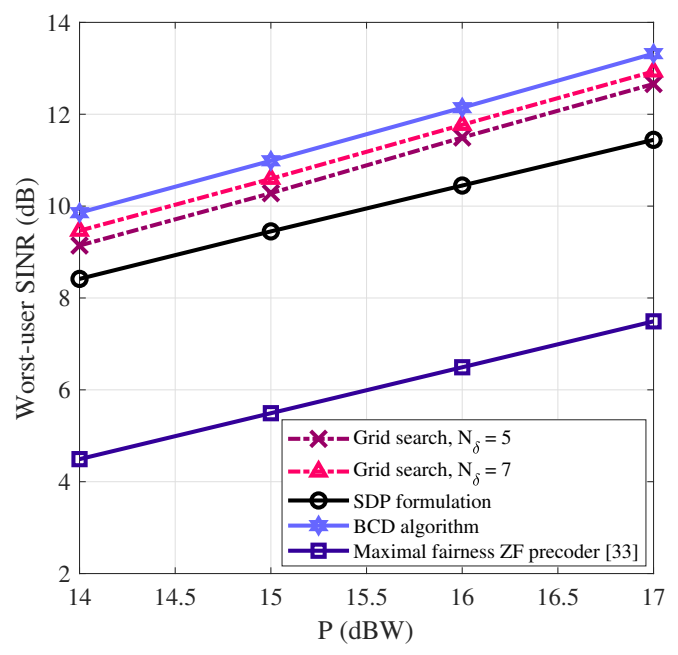

Fig. 8. Minimum SINR among $K=4$ users versus total power constraint for the optimized 8 -ary constellation.

Figures 7-9 show the optimized worst-user SINR obtained via different SLP SINR balancing approaches for three constellations 8-PSK, optimized 8-ary and 16-QAM, respectively. We also compare the results with those of the maximal fairness zero-forcing precoder [33], and the bisection algorithm [18]. The method based on gird search is used here as a basis for comparison. We choose $N_{\delta}=5$ and $N_{\delta}=7$ points to search over the interval $[0,2.5]$. The SDP formulation, while being always superior to the maximal fairness ZF precoding by at least $1 \mathrm{~dB}$, is a lower bound on the optimal solution of SLP max-min SINR. The BCD algorithm, on the other hand, provides gains up to $2 \mathrm{~dB}$ with respect to the SDP formulation for the optimized 8-ary constellation. Furthermore, Fig. 8 shows that this iterative method is able to achieve even better solutions than the grid search with $N_{\delta}=7$, having an extremely lower complexity.

In Fig. 10, the optimized worst-user SINR is plotted as a function of system dimension, where the users' symbols are 


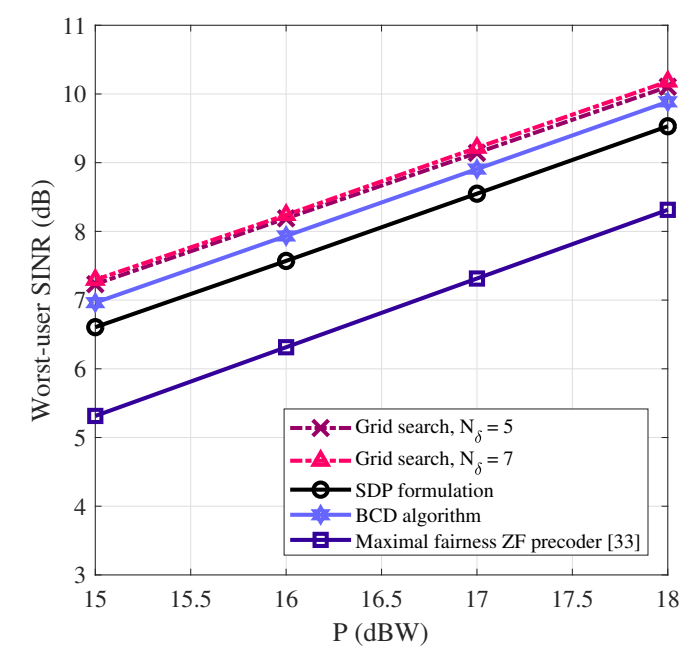

Fig. 9. Minimum SINR among $K=4$ users versus total power constraint for 16-QAM constellation.

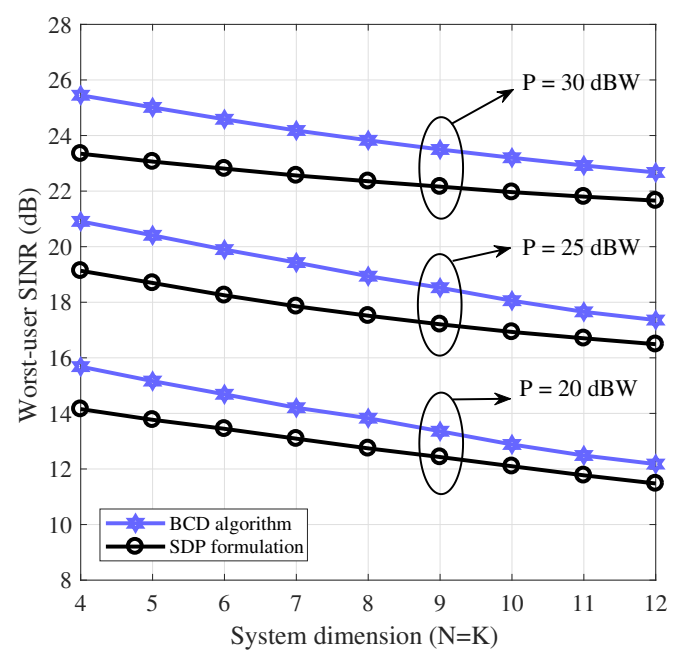

Fig. 10. The worst-user SINR as a function of system dimension for different values of total power budget.

taken from the optimized 8-ary constellation. As expected, a lower minimum SINR is achieved with increasing the system dimension; however, SINR drops more gradually with respect to the system dimension for larger power budgets.

In Fig. 11, we compare the convergence rate of the $\mathrm{BCD}$ algorithm versus system dimension for different power budgets with 8-PSK and the optimized 8-ary constellation. Here, the convergence rate is expressed in terms of the average number of iterations until the terminating condition is met. It can be seen that the BCD algorithm solving the SLP max-min SINR for $N=K=4$ converges after a few iterations with an average of up to 6 iterations for $P=30 \mathrm{~dB}$, where each iteration consists of a single SDP. Figure 11 also shows a slightly slower convergence behavior for higher values of $P$ which is due to a larger feasible region. Furthermore, in order to evaluate the dependence of the convergence behavior on the constellation size, in Fig. 12 we plot the number of iterations

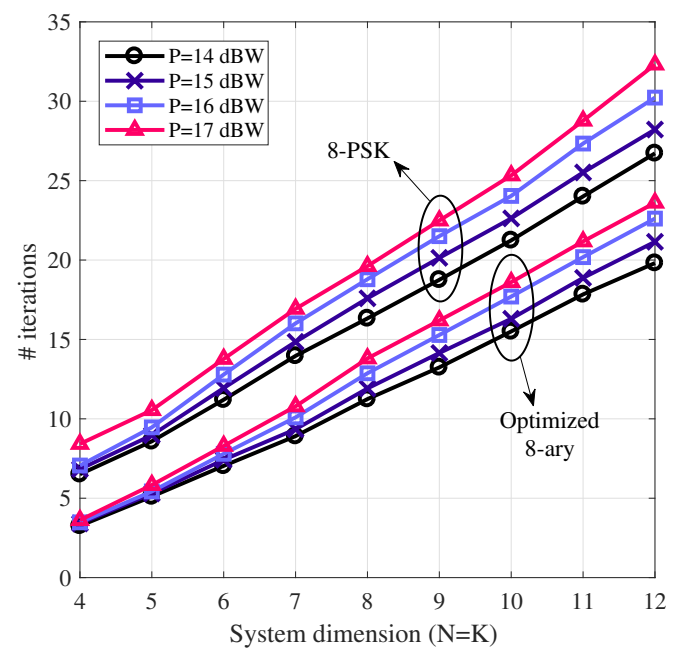

Fig. 11. Number of iterations until convergence of the BCD algorithm as a function of system dimension for different values of total power budget.

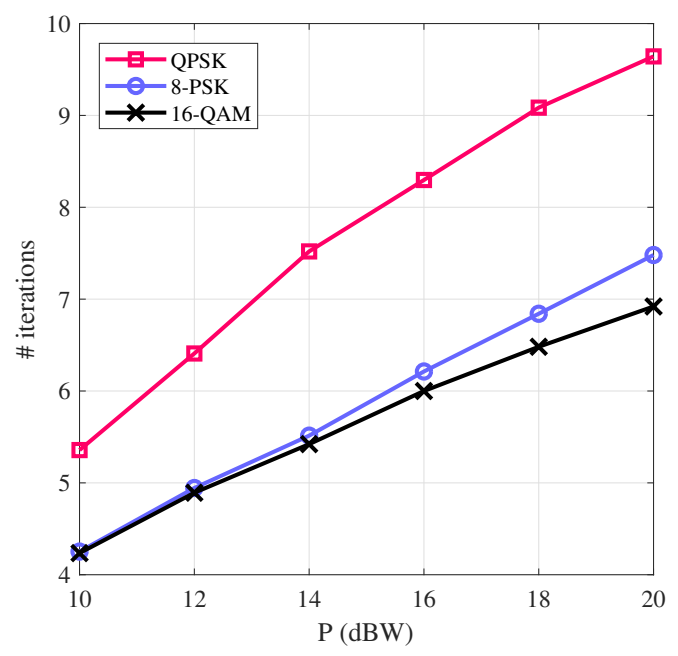

Fig. 12. Number of iterations until convergence of the $\mathrm{BCD}$ algorithm as a function of total power budget for three constellations with different orders.

for three modulation schemes with different orders. It can be inferred from Fig. 12 that for a constellation set with narrower (unbounded) DPCIRs, the BCD algorithm needs more iterations to converge. In other words, as the angle between the two distance preserving boundaries becomes smaller, the two block coordinates $\boldsymbol{\delta}_{\mathcal{K}, 1}$ and $\boldsymbol{\delta}_{\mathcal{K}, 2}$ will be more aligned, resulting in fewer coordinate recursions. The DPCIR angles are equal to $\pi / 2$ and $\pi / 4$ for QPSK and 8-PSK, respectively. Complexity comparison between SDP and BCD: The SDP formulation needs to solve a single convex problem per symbol time. On the other hand, according to Fig. 11, the BCD algorithm converges after 4 iterations (optimized 8-ary) and 6-8 iterations (8-PSK), on average, where each iteration involves solving one SDP. The BCD algorithm, though having a higher complexity than the SDP formulation, offers gains of 1.5-2.0 dB (optimized 8-ary) and 0.2-0.4 dB (8-PSK) in the worst-user SINR (see Fig. 7 and Fig. 8). Therefore, the 
BCD algorithm provides a reasonable complexity-performance tradeoff with respect to the SDP formulation. In order to summarize and compare the complexities of the two methods, in Table I we present the problem size (in terms of the number of optimization variables) and the number of iterations per symbol time for each method.

TABLE I

COMPLEXITIES OF THE PROPOSED ALTERNATIVE METHODS FOR SLP MAX-MIN SINR.

\begin{tabular}{ccc}
\hline Method & Problem size & Iteration/symbol time \\
\hline SDP formulation & $2 N+2 K+1$ & 1 \\
\hline BCD algorithm & $2 N+2 K-|\mathcal{K}|+1$ & Fig. 11 and Fig. 12 \\
\hline
\end{tabular}

\section{CONCLUSION}

In this paper, we addressed two well-known precoding design problems in a downlink multiuser (unicast) channel, namely, power optimization and SINR balancing, on a symbollevel basis. CIRs are the key to formulate the SLP problem as they define the constraints to achieve CI at the receiver of each user. We considered a general category of CIRs named as DPCIR. Full characterization of DPCIRs for a generic constellation was provided which led to extracting various properties for these regions. Using a systematic description for DPCIRs, we formulated and discussed the SLP optimization problems. The SINR-constrained SLP power minimization was investigated in a realistic scenario and a simple feasibility condition was derived. We also expressed this optimization in an equivalent form with a reduced problem size. For the more challenging and generally non-convex problem of SLP SINR balancing under max-min fairness criterion, the properties of DPCIRs helped us to reformulate the problem in a (sub-optimal) convex form. Subsequently, to tackle this problem, two alternative approaches were proposed, namely, SDP formulation and BCD optimization. Finally, we provided a detailed comparison of complexity for the proposed methods.

\section{ACKNOWLEDGMENT}

The authors are supported by the Luxembourg $\mathrm{Na}$ tional Research Fund (FNR) under CORE Junior project: C16/IS/11332341 Enhanced Signal Space opTImization for satellite comMunication Systems (ESSTIMS).

\section{APPENDIX A}

\section{PROOF OF LEMMA 2}

The intersection of a finite number of closed halfspaces is an unbounded polyhedron if and only if the outward normals to the associated boundary hyperplanes lie on a single closed halfspace [38, p. 20, Theorem 4]. Accordingly, for any $\mathbf{x}_{i} \in \mathcal{X}$ with unbounded $\mathcal{D}_{i, \mathrm{ML}}$, all the outward normal vectors $-\mathbf{a}_{i, j}, j \in \mathcal{J}_{i}$ lie on a single halfspace. Since the polyhedron $\mathcal{D}_{i, \mathrm{DP}}$ has the same set of outward normals $-\mathbf{a}_{i, j}, j \in \mathcal{J}_{i}$, it is also unbounded. An unbounded polyhedron is uniquely determined from its vertices and the directions of its infinite edges [38, p. 31, Theorem 4]. Furthermore, it is straightforward to check that $\mathbf{x}_{i}$ is the unique solution of $\mathbf{A}_{i} \mathbf{x}=\mathbf{b}_{i}+\mathbf{c}_{i \text {,DP }}$, i.e., all the contributing hyperplanes have a common intersection point $\mathbf{x}_{i}$. This means that $\mathcal{D}_{i, \mathrm{DP}}$, which is given by the solution set of $\mathbf{A}_{i} \mathbf{x} \succeq \mathbf{b}_{i}+\mathbf{c}_{i \text {,DP }}$, has a single vertex at $\mathbf{x}_{i}$ and two infinite edges, i.e., a polyhedral angle. In addition, since any two neighboring points share a common Voronoi edge, the two infinite edges of $\mathcal{D}_{i \text {,DP }}$ correspond to the two neighboring points of $\mathbf{x}_{i}$ on $\mathbf{b d} \mathcal{X}$ (i.e., $\mathcal{S}_{i} \cap \mathbf{b d} \mathcal{X}$ ) with unbounded Voronoi regions. Each infinite edge of $\mathcal{D}_{i, \mathrm{DP}}$ is then parallel to a hyperplane with normal vector $\mathbf{a}_{i, j}=\mathbf{x}_{i}-\mathbf{x}_{j}$, where $\mathbf{x}_{j} \in \mathcal{S}_{i} \cap \mathbf{b d} \mathcal{X}$; therefore it is perpendicular to $\mathbf{x}_{i}-\mathbf{x}_{j}$. This completes the proof.

\section{APPENDIX B \\ PROOF OF LEMMA 3}

In order to prove this lemma, we first state a well-known property of convex sets.

Property 2. $\mathbf{v}_{o}$ is the minimum distance vector from the origin to the convex set $\mathcal{V}$ iff for any vector $\mathbf{v} \in \mathcal{V}$ we have $\mathbf{v}_{o}^{\mathrm{T}} \mathbf{v} \geq$ $\mathbf{v}_{o}^{\mathrm{T}} \mathbf{v}_{o}$, with equality for $\mathbf{v}$ lying on the hyperplane orthogonal to $\mathbf{v}_{o}[39$, p. 69, Theorem 1].

For any $\mathbf{x}_{i} \in \operatorname{int} \mathcal{X}$, Lemma 3 holds straightforwardly as $\mathcal{D}_{i, \mathrm{DP}}=\mathbf{x}_{i}$. Therefore, in what follows we only focus on the constellation points belonging to $\operatorname{bd} \mathcal{X}$.

Sufficiency: Having $\mathbf{0} \in \operatorname{conv} \mathcal{X}$, let further assume that $\mathbf{0} \in$ $\mathcal{X}$. This assumption, as mentioned earlier in section III, does not have any impact on $\mathcal{D}_{i, \mathrm{DP}}$ for any $\mathbf{x}_{i} \in \mathbf{b d} \mathcal{X}$, regardless of whether $\mathbf{0} \in \mathbf{b d} \mathcal{X}$ or $\mathbf{0} \in \operatorname{int} \mathcal{X}$. By substituting $\mathbf{x}_{j}=\mathbf{0}$ in (20), for all $\mathbf{x}_{i} \in \mathcal{X}$ we have $\|\mathbf{x}\| \geq\left\|\mathbf{x}_{i}\right\|, \forall \mathbf{x} \in \mathcal{D}_{i, \mathrm{DP}}$. This completes the proof of sufficiency.

Necessity: By contradiction, if $\mathbf{0} \notin \operatorname{conv} \mathcal{X}$, let assume a new constellation set $\tilde{\mathcal{X}}$ having all the points of $\mathcal{X}$ including the origin, i.e., $\tilde{\mathcal{X}}=\mathcal{X} \cup\{\mathbf{0}\}$, hence $\operatorname{conv} \mathcal{X} \subset \operatorname{conv} \tilde{\mathcal{X}}$. Clearly, $\mathbf{0} \in \mathbf{b d} \tilde{\mathcal{X}}$ and according to Lemma 2 , there always exist exactly two constellation points on bd $\tilde{\mathcal{X}}$ that $\mathbf{0}$ contributes to their DPCIRs. Suppose $\mathbf{x}_{l}$ be one of these points with $\mathcal{D}_{l, \mathrm{DP}}$ and $\tilde{\mathcal{D}}_{l, \mathrm{DP}}$ denoting its associated DPCIR relative to $\mathcal{X}$ and $\tilde{\mathcal{X}}$, repectively. We denote by $\tilde{\mathcal{S}}_{l}$ the set of neighboring points of $\mathbf{x}_{l}$ in $\tilde{\mathcal{X}}$. Let $\mathcal{H}_{l, o}=\left\{\mathbf{x} \mid \mathbf{x} \in \mathbb{R}^{2}, \mathbf{x}_{l}^{\mathrm{T}} \mathbf{x} \geq \mathbf{x}_{l}^{\mathrm{T}} \mathbf{x}_{l}\right\}$ be the distance preserving halfspace from $\mathbf{0}$ to $\mathbf{x}_{l}$. Since $\mathbf{0} \in \tilde{\mathcal{S}}_{l}$, we have $\tilde{\mathcal{D}}_{l, \mathrm{DP}}=\mathcal{H}_{l, o} \cap \mathcal{D}_{l, \mathrm{DP}} \neq \mathcal{D}_{l, \mathrm{DP}}$, i.e., the halfspace $\mathcal{H}_{l, o}$ does not contain $\mathcal{D}_{l, \mathrm{DP}}$. Hence, $\left\{\mathbf{x} \mid \mathbf{x} \in \mathbb{R}^{2}, \mathbf{x}_{l}^{\mathrm{T}} \mathbf{x}=\mathbf{x}_{l}^{\mathrm{T}} \mathbf{x}_{l}\right\}$ is not a supporting hyperplane for $\mathcal{D}_{l, \mathrm{DP}}$ at $\mathbf{x}_{l}[29, \mathrm{p}$. 51]. This implies that there exist some $\mathbf{x} \in \mathcal{D}_{l, \mathrm{DP}}$ for which $\mathbf{x}_{l}^{\mathrm{T}} \mathbf{x}<\mathbf{x}_{l}^{\mathrm{T}} \mathbf{x}_{l}$. According to Property 2 (which gives a necessary and sufficient condition), $\mathbf{x}_{l}$ is not the minimum distance vector from the origin in $\mathcal{D}_{l, \mathrm{DP}}$. Consequently, $\|\mathbf{x}\| \geq\left\|\mathbf{x}_{l}\right\|$ does not hold for some $\mathbf{x} \in \mathcal{D}_{l, \mathrm{DP}}$ which contradicts $\|\mathbf{x}\| \geq\left\|\mathbf{x}_{l}\right\|, \forall \mathbf{x} \in \mathcal{D}_{l, \mathrm{DP}}$.

\section{APPENDIX C \\ PROOF OF THEOREM 1}

In order to prove this theorem we need the following lemma.

Lemma 5. If $\mathbf{0} \notin \operatorname{conv} \mathcal{X}$, there exists at least one constellation point $\mathbf{x}_{l} \in \mathcal{X}$ for which for any $\mathbf{x} \in \mathcal{D}_{l, D P}$, $\mathbf{0} \notin \operatorname{conv} \tilde{\mathcal{X}}_{\mathbf{x}_{l}, \mathbf{x}}$, where $\tilde{\mathcal{X}}_{\mathbf{x}_{l}, \mathbf{x}}=\mathcal{X} \cup\{\mathbf{x}\}$. 
Proof: If $\mathbf{0} \notin \operatorname{conv} \mathcal{X}$, for any $\mathbf{x}_{i} \in \mathcal{X}$ and any $\mathbf{x} \in \mathcal{D}_{i, \mathrm{DP}}$ with $\tilde{\mathcal{X}}_{\mathbf{x}_{i}, \mathbf{x}}=\mathcal{X} \cup\{\mathbf{x}\}$, let define $\mathcal{C}_{i}=\bigcup_{\mathbf{x} \in \mathcal{D}_{i, \mathrm{DP}}} \operatorname{conv} \tilde{\mathcal{X}}_{\mathbf{x}_{i}, \mathbf{x}}$. Having conv $\mathcal{X} \subseteq \operatorname{conv} \tilde{\mathcal{X}}_{\mathbf{x}_{i}, \mathbf{x}}$, it follows from the definition of convex hull that $\operatorname{conv} \mathcal{X}=\bigcap_{\mathbf{x}_{i} \in \mathcal{X}} \mathcal{C}_{i}$. If $\mathbf{0} \in \mathcal{C}_{i}, \forall \mathbf{x}_{i} \in \mathcal{X}$, then $\mathbf{0} \in \operatorname{conv} \mathcal{X}$ which contradicts our assumption. Hence there must exist at least one constellation point, say $\mathbf{x}_{l}$, for which $\mathcal{C}_{l}$ and therefore none of $\operatorname{conv} \tilde{\mathcal{X}}_{\mathbf{x}_{l}, \mathbf{x}}, \forall \mathbf{x} \in \mathcal{D}_{l, \mathrm{DP}}$ contains the origin, as required.

Now, we can complete the proof of Theorem 1 as follows. Sufficiency: Suppose $\mathbf{0} \in \operatorname{conv} \mathcal{X}$. Assuming a constellation point $\mathbf{x}_{i} \in \mathcal{X}$ and its DPCIR $\mathcal{D}_{i, \mathrm{DP}}$, let $\mathbf{y}_{1}$ and $\mathbf{y}_{2}$ be two points in $\mathcal{D}_{i, \mathrm{DP}}$ such that $\mathbf{A}_{i} \mathbf{y}_{1}=\mathbf{b}_{i}+\mathbf{c}_{i, \mathrm{DP}}+\boldsymbol{\delta}_{i, 1}$ and $\mathbf{A}_{i} \mathbf{y}_{2}=$ $\mathbf{b}_{i}+\mathbf{c}_{i, \mathrm{DP}}+\boldsymbol{\delta}_{i, 2}$ with $\boldsymbol{\delta}_{i, 1}, \boldsymbol{\delta}_{i, 2} \in \mathbb{R}_{+}^{M_{i}}$ and $\boldsymbol{\delta}_{i, 1} \prec \boldsymbol{\delta}_{i, 2}$. Let consider a new constellation $\tilde{\mathcal{X}}=\mathcal{X} \cup\left\{\mathbf{y}_{1}\right\}$. It is clear that $\operatorname{conv} \mathcal{X} \subseteq \operatorname{conv} \tilde{\mathcal{X}}$, and therefore $\mathbf{0} \in \operatorname{conv} \tilde{\mathcal{X}}$. The DPCIR of $\mathbf{y}_{1}$ can be described as $\mathcal{D}_{\mathbf{y}_{1}, \mathrm{DP}}=\left\{\mathbf{x} \mid \mathbf{x} \in \mathbb{R}^{2}, \mathbf{A}_{i} \mathbf{x}=\right.$ $\left.\mathbf{b}_{i}+\mathbf{c}_{i, \mathrm{DP}}+\boldsymbol{\delta}_{i, 1}+\boldsymbol{\delta}_{1}, \boldsymbol{\delta}_{1} \in \mathbb{R}_{+}^{M_{i}}\right\}$. Let $\overline{\boldsymbol{\delta}}=\boldsymbol{\delta}_{i, 2}-\boldsymbol{\delta}_{i, 1}$, then $\mathbf{A}_{i} \mathbf{y}_{2}=\mathbf{b}_{i}+\mathbf{c}_{i, \mathrm{DP}}+\boldsymbol{\delta}_{i, 1}+\overline{\boldsymbol{\delta}}, \overline{\boldsymbol{\delta}} \in \mathbb{R}_{++}^{M_{i}}$, which means that $\mathbf{y}_{2} \in \mathcal{D}_{\mathbf{y}_{1}, \mathrm{DP}}$. As a consequence, from Lemma 3 , we have $\left\|\mathbf{y}_{1}\right\|<\left\|\mathbf{y}_{2}\right\|$ and the proof of sufficiency is complete.

Necessity: By contradiction, suppose $0 \notin \operatorname{conv} \mathcal{X}$. Then, based on Lemma 5 , there exists a constellation point $\mathbf{x}_{l}$ for which $\mathbf{0} \notin \operatorname{conv} \tilde{\mathcal{X}}_{\mathbf{x}_{l}, \mathbf{x}}, \forall \mathbf{x} \in \mathcal{D}_{l, \text { Dp }}$. Let $\mathbf{y}_{1} \in \mathcal{D}_{l, \text { DP }}$, then $\mathbf{A}_{l} \mathbf{y}_{1}=\mathbf{b}_{l}+\mathbf{c}_{l, \mathrm{DP}}+\boldsymbol{\delta}_{l, 1}$ with $\boldsymbol{\delta}_{l, 1} \in \mathbb{R}_{+}^{M_{l}}$. The DPCIR associated with $\mathbf{y}_{1}$ can be expressed as $\mathcal{D}_{\mathbf{y}_{1}, \mathrm{DP}}=\{\mathbf{x} \mid$ $\left.\mathbf{x} \in \mathbb{R}^{2}, \mathbf{A}_{l} \mathbf{x}=\mathbf{b}_{l}+\mathbf{c}_{l, \mathrm{DP}}+\boldsymbol{\delta}_{l, 1}+\boldsymbol{\delta}_{1}, \boldsymbol{\delta}_{1} \in \mathbb{R}_{+}^{M_{l}}\right\}$. Since $\mathbf{0} \notin \operatorname{conv} \tilde{\mathcal{X}}_{\mathbf{x}_{l}, \mathbf{y}_{1}}$, it follows from Lemma 3 and Property 2 that there exists $\mathbf{y}_{2} \in \mathcal{D}_{\mathbf{y}_{1}, \mathrm{DP}}$ such that $\mathbf{A}_{l} \mathbf{y}_{2}=\mathbf{b}_{l}+\mathbf{c}_{l, \mathrm{DP}}+$ $\boldsymbol{\delta}_{l, 1}+\overline{\boldsymbol{\delta}}, \overline{\boldsymbol{\delta}} \in \mathbb{R}_{++}^{M_{l}}$, for which $\left\|\mathbf{y}_{2}\right\|<\left\|\mathbf{y}_{1}\right\|$. But $\boldsymbol{\delta}_{l, 1}+\overline{\boldsymbol{\delta}}=\boldsymbol{\delta}_{l, 2}$ yields $\boldsymbol{\delta}_{l, 2} \succ \boldsymbol{\delta}_{l, 1}$ which is a contradiction. This completes the proof.

\section{REFERENCES}

[1] M. Bengtsson and B. Ottersten, Handbook of Antennas in Wireless Communications, 2001, ch. Optimal and suboptimal transmit beamforming.

[2] A. B. Gershman, N. D. Sidiropoulos, S. Shahbazpanahi, M. Bengtsson, and B. Ottersten, "Convex optimization-based beamforming," IEEE Signal Process. Mag., vol. 27, no. 3, pp. 62-75, May 2010.

[3] E. Björnson, M. Bengtsson, and B. Ottersten, "Optimal multiuser transmit beamforming: A difficult problem with a simple solution structure," IEEE Signal Process. Mag., vol. 31, no. 4, pp. 142-148, Jul. 2014.

[4] Y. F. Liu, Y. H. Dai, and Z. Q. Luo, "Coordinated beamforming for MISO interference channel: Complexity analysis and efficient algorithms," IEEE Trans. Signal Process., vol. 59, no. 3, pp. 1142-1157, Mar. 2011.

[5] N. D. Sidiropoulos, T. N. Davidson, and Z.-Q. Luo, "Transmit beamforming for physical-layer multicasting," IEEE Trans. Signal Process., vol. 54, no. 6, pp. 2239-2251, Jun. 2006.

[6] A. Wiesel, Y. C. Eldar, and S. Shamai, "Linear precoding via conic optimization for fixed MIMO receivers," IEEE Trans. Signal Process., vol. 54, no. 1, pp. 161-176, Jan. 2006.

[7] O. Tervo, L. N. Tran, and M. Juntti, "Optimal energy-efficient transmit beamforming for multi-user MISO downlink," IEEE Trans. Signal Process., vol. 63, no. 20, pp. 5574-5588, Oct. 2015.

[8] E. Visotsky and U. Madhow, "Optimum beamforming using transmit antenna arrays," in 1999 IEEE 49th Vehicular Technology Conference (Cat. No.99CH36363), vol. 1, Jul. 1999, pp. 851-856 vol.1.

[9] M. Schubert and H. Boche, "Solution of the multiuser downlink beamforming problem with individual SINR constraints," IEEE Trans. Veh. Technol., vol. 53, no. 1, pp. 18-28, Jan. 2004.

[10] _ , "Iterative multiuser uplink and downlink beamforming under SINR constraints," IEEE Trans. Signal Process., vol. 53, no. 7, pp. 2324 2334, Jul. 2005
[11] S. S. Christensen, R. Agarwal, E. D. Carvalho, and J. M. Cioffi, "Weighted sum-rate maximization using weighted MMSE for MIMOBC beamforming design," IEEE Trans. Wirel. Commun., vol. 7, no. 12, pp. 4792-4799, Dec. 2008.

[12] M. Stojnic, H. Vikalo, and B. Hassibi, "Rate maximization in multiantenna broadcast channels with linear preprocessing," IEEE Trans. Wirel. Commun., vol. 5, no. 9, pp. 2338-2342, Sep. 2006.

[13] E. Biglieri, R. Calderbank, A. Constantinides, A. Goldsmith, A. Paulraj, and $\mathrm{H}$. V. Poor, MIMO wireless communications. Cambridge university press, 2007.

[14] C. Masouros and E. Alsusa, "Soft linear precoding for the downlink of DS/CDMA communication systems," IEEE Trans. Veh. Technol., vol. 59, no. 1, pp. 203-215, Jan. 2010.

[15] C. Masouros, T. Ratnarajah, M. Sellathurai, C. B. Papadias, and A. K. Shukla, "Known interference in the cellular downlink: a performance limiting factor or a source of green signal power?" IEEE Commun. Mag., vol. 51 , no. 10 , pp. $162-171$, Oct. 2013.

[16] C. Masouros, "Correlation rotation linear precoding for MIMO broadcast communications," IEEE Trans. Signal Process., vol. 59, no. 1, pp. 252262, Jan. 2011

[17] C. Masouros and G. Zheng, "Exploiting known interference as green signal power for downlink beamforming optimization," IEEE Trans. Signal Process., vol. 63, no. 14, pp. 3628-3640, Jul. 2015.

[18] M. Alodeh, S. Chatzinotas, and B. Ottersten, "Constructive multiuser interference in symbol level precoding for the MISO downlink channel," IEEE Trans. Signal Process., vol. 63, no. 9, pp. 2239-2252, May 2015.

[19] P. V. Amadori and C. Masouros, "Constant envelope precoding by interference exploitation in phase shift keying-modulated multiuser transmission," IEEE Trans. Wirel. Commun., vol. 16, no. 1, pp. 538550, Jan. 2017.

[20] A. Kalantari, M. Soltanalian, S. Maleki, S. Chatzinotas, and B. Ottersten, "Directional modulation via symbol-level precoding: A way to enhance security," IEEE J. Sel. Topics in Signal Process., vol. 10, no. 8, pp. 1478-1493, Dec. 2016.

[21] M. Alodeh, S. Chatzinotas, and B. Ottersten, "Symbol-level multiuser MISO precoding for multi-level adaptive modulation," IEEE Trans. Wirel. Commun., vol. 16, no. 8, pp. 5511-5524, Aug. 2017.

[22] A. Li and C. Masouros, "Exploiting constructive mutual coupling in P2P MIMO by analog-digital phase alignment," IEEE Trans. Wirel. Commun., vol. 16, no. 3, pp. 1948-1962, Mar. 2017.

[23] M. Alodeh, D. Spano, S. Chatzinotas, and B. Ottersten, "Faster-thannyquist spatiotemporal symbol-level precoding in the downlink of multiuser MISO channels," in IEEE Int. Conf. on Acoustics, Speech and Signal Processing (ICASSP), Mar. 2017, pp. 3779-3783.

[24] D. Spano, M. Alodeh, S. Chatzinotas, and B. Ottersten, "Per-antenna power minimization in symbol-level precoding," in IEEE Global Communications Conf. (GLOBECOM), Dec. 2016, pp. 1-6.

[25] A. Haqiqatnejad, F. Kayhan, and B. Ottersten, "Constructive interference for generic constellations," IEEE Signal Process. Lett., vol. 25, no. 4, pp. 586-590, Apr. 2018.

[26] T. H. Chang, Z. Q. Luo, and C. Y. Chi, "Approximation bounds for semidefinite relaxation of max-min-fair multicast transmit beamforming problem," IEEE Trans. Signal Process., vol. 56, no. 8, pp. 3932-3943, Aug. 2008.

[27] F. Shu, X. Wu, J. Li, R. Chen, and B. Vucetic, "Robust synthesis scheme for secure multi-beam directional modulation in broadcasting systems," IEEE Access, vol. 4, pp. 6614-6623, 2016.

[28] A. Haqiqatnejad, F. Kayhan, and B. Ottersten, "Symbol-level precoding design for max-min SINR in multiuser MISO broadcast channels," arXiv:1803.06871 [cs.IT], Mar. 2018.

[29] S. Boyd and L. Vandenberghe, Convex Optimization. Cambridge Univ. Press, 2004

[30] F. Kayhan and G. Montorsi, "Constellation design for memoryless phase noise channels," IEEE Trans. Wirel. Commun., vol. 13, no. 5, pp. 2874 2883, May 2014.

[31] F. Aurenhammer and R. Klein, Handbook of computational geometry, 2000, ch. Voronoi diagrams, pp. 201-290.

[32] M. X. Goemans, "Linear programming and polyhedral combinatorics," 2009.

[33] A. Wiesel, Y. C. Eldar, and S. Shamai, "Zero-forcing precoding and generalized inverses," IEEE Trans. Signal Process., vol. 56, no. 9, pp. 4409-4418, Sep. 2008.

[34] R. M. Freund, "Solution methods for quadratic optimization," 2004.

[35] R. Bro and S. De Jong, "A fast non-negativity-constrained least squares algorithm," J. Chemometrics: A Journal of the Chemometrics Society, vol. 11 , no. 5 , pp. $393-401,1997$. 
[36] D. P. Bertsekas, Nonlinear Programming. Athena scientific Belmont, 1999.

[37] Digital Video Broadcasting (DVB) Part 2: DVB-S2 Extensions (DVBS2X). ETSI EN Std. 302 307-2 V1.1.1, 2014.

[38] A. D. Alexandrov, Convex Polyhedra. Springer, 2005.

[39] D. G. Luenberger, Optimization by Vector Space Methods. New York: Wiley, 1988 